\title{
Quantum interference in two-photon excitation with squeezed and coherent fields
}

\author{
N. Ph. Georgiades, E. S. Polzik, * and H. J. Kimble \\ Norman Bridge Laboratory of Physics, 12-33 California Institute of Technology, Pasadena, California 91125
}

(Received 30 December 1997)

\begin{abstract}
Two-photon excitation of a three-level atom in a ladder configuration $(1 \rightarrow 2 \rightarrow 3)$ by simultaneous illumination with fields in squeezed vacuum and coherent states results in quantum interference for the excitation process. The particular configuration considered here is one for which the signal and idler output fields of a subthreshold nondegenerate optical parametric oscillator are in resonance with the two-stepwise dipole atomic transitions $(1 \rightarrow 2,2 \rightarrow 3)$, while a "reference oscillator" field is in two-photon resonance with the quadrupole transition $(1 \rightarrow 3)$. In an extension of the work of Ficek and Drummond [Phys. Rev. A 43, 6247 (1991)], a theoretical formulation based on the full quantum master equation for the problem is presented. The combined effects of quantum interference and the nonclassical character of the squeezed state are investigated, and offer the potential for a new detection strategy for quantum fluctuations of the electromagnetic field with ultrahigh frequencies (10's-100's THz). Based on the theory developed, we analyze quantum interference in excitation in several special cases relevant to experimental realizations, including the effects of a small focusing angle of the squeezing onto the atoms, and unusual population inversions. Special emphasis is given to identifying intrinsically quantum optical field effects versus classical field effects. Procedures that could distinguish between the two (i.e., classical and nonclassical) are suggested. [S1050-2947(98)06109-5]
\end{abstract}

PACS number(s): 42.50.Ct, 42.50.Dv, 32.80.Wr, 42.50.Hz

\section{INTRODUCTION}

The interaction of squeezed light with atoms has been extensively studied theoretically over the past decade [1,2]. The phenomena revealed by these studies can be broadly divided into two categories, according to the efficiency with which the squeezed field and an atom are coupled. In the first case, efficient coupling of atoms to the nonclassical reservoir provided by the squeezed fields leads to a modification of the radiative processes of the atoms, such as modified atomic decay rates and altered atomic saturation. In the second case of inefficient coupling, perhaps surprisingly, the manifestly quantum fluctuations of the squeezed state can nevertheless drive atomic populations in ways not possible if the statistics of the fields were classical. Examples of the latter case are two-photon excitation [3-5], photon statistics in resonance fluorescence $[6,7]$, and squeezing of the collective atomic spin [27]. In contrast to the numerous theoretical advances in the area, experimental work has been proven to be extremely difficult with only one experiment [8] having demonstrated a purely nonclassical effect of the second category. In particular, in our experiment of Ref. [8], a two-photon transition in a three-level atom $(1 \rightarrow 2 \rightarrow 3)$ was excited by the correlated signal and idler fields of a subthreshold nondegenerate optical parametric oscillator. In striking contrast to the classical quadratic dependance, the excited-state population $\rho_{33}$ was observed to exhibit a slope less than 2 with respect to the incident intensity; in fact observations with a slope as low as 1.3 were recorded. An additional attempt in our group to couple squeezed light and atoms within the setting of cavity QED [9] was sensitive only to the fact that the squeezed states have an asymmetric phase-space distribution for the fluctuations of the quadrature-field amplitudes, but did not

\footnotetext{
*Permanent address: Institute of Physics and Astronomy, Aarhus University, Aarhus C DK-8000, Denmark.
}

convincingly demonstrate a sensitivity to the fact that the excitation field was nonclassical.

To investigate two-photon excitation with squeezed light further, we carried out yet another experiment beyond that described in Ref. [8]. Here the objective was to explore explicitly the issue of the phase sensitivity of the excitation process, where now the quadrature amplitudes $X_{\theta}(\Omega)$, defined by

$$
\hat{X}_{\theta}(\Omega) \equiv \hat{a}(\Omega) e^{-i \theta}+\hat{a}^{\dagger}(\Omega) e^{i \theta},
$$

have appreciable Fourier content for signal $\omega_{s}$ and idler $\omega_{i}$ frequencies $\omega_{s, i}=\omega_{0} \pm \Omega_{0}$ offset by $\Omega_{0} / 2 \pi \simeq 12.5 \mathrm{THz}$ from the center frequency $\omega_{0}$. This frequency span is well beyond the detection capabilities of conventional homodyne photodetection schemes, so that a new technique is required for investigation of such high frequency (cw) correlations. The whole approach in Ref. [10] is an attempt to develop such a new detection strategy, whereby the atom itself is employed as a nonlinear mixing element for the demodulation of the high-frequency fluctuations of the field.

Briefly reviewing the principles of the experiment of Ref. [10], we recall that it is based on the combination of twophoton nonclassical excitation as studied in Ref. [8], together with coherent excitation leading to quantum interference (QI) as described in Ref. [12]. More specifically, the sequence $1 \rightarrow 2 \rightarrow 3$ corresponding to the $6 S_{1 / 2} F=4$ $\rightarrow 6 P_{3 / 2} F^{\prime}=5 \rightarrow 6 D_{5 / 2} F^{\prime \prime}=6$ transition in atomic Cs was excited by the signal and idler output fields from a subthreshold nondegenerate optical parametric oscillator (ND-OPO) at frequencies $\left(\omega_{s}, \omega_{i}\right)$ corresponding to the resonance frequencies $\left(\omega_{21}, \omega_{32}\right)$ of the $6 S_{1 / 2} F=4 \rightarrow 6 P_{3 / 2} F^{\prime}=5$ and $6 P_{3 / 2} F^{\prime}=5 \rightarrow 6 D_{5 / 2} F^{\prime \prime}=6$ transitions, respectively. At the same time, the atom was illuminated with a coherent "reference oscillator', (RO) field of frequency $\omega_{\mathrm{RO}}$, resonant with the two-photon transition frequency corresponding to half that of the $6 S_{1 / 2} F=4 \rightarrow 6 D_{5 / 2} F^{\prime \prime}=6$ transition, $\omega_{\mathrm{RO}} \simeq \omega_{31} / 2$. 
Hence, the excited state 3 could be reached via two pathways, i.e., either via two dipole stepwise absorptions from the signal and idler fields, resulting in the $1 \rightarrow 2$ and $2 \rightarrow 3$ transitions, respectively, or via quadrupole, two-photon absorption from the RO field, which leads to the direct $1 \rightarrow 3$ transition. The amplitudes for these two indistinguishable excitation pathways interfere coherently to result in QI which can manifest itself as a modulation of the excited-state population $\rho_{33}$ as the phases of the various excitation fields are varied. As observed and analyzed in Ref. [10], this effect can lead to phase-sensitive detection of squeezing for signal and idler fields separated by hundreds of THz, where in Ref. [10] the correlated signal and idler fields were separated by 25 THz. Implicit in this discussion is that the excitation fields are phase coherent over the relevant relaxation time $T$ over the other which is a necessary condition for the preservation of QI. If the excitation lasers were incoherent with each other over $T$, the total excitation probability would simply be the incoherent sum of the excitation probabilities of each pathway of excitation.

In the present paper, our goal is to extend the simple theoretical picture given in Ref. [10], and to provide a general theoretical foundation for these types of experiments. We also wish to explore possible extensions of our work, with emphasis on the nonclassical properties of the squeezed fields in contrast to our previous theoretical analysis in Ref. [11] which deals with all-coherent-state excitation. To this end, in Sec. II we describe in more detail the radiation source and atomic system under consideration, and then in Sec. III present the Hamiltonian formulation of our problem. By drawing heavily on the prior analysis of Ref. [5], in Sec. IV we derive the master equation for the atom illuminated with signal, idler, and reference oscillator fields, from which the Bloch equations that determine the dynamical evolution of the system follow in Sec. V. These equations are solved for the atomic populations in steady state in Sec. VI. Section VII is devoted to a discussion of the effects of a small solid angle over which the squeezed fields are focused onto the atom. Section VIII analyzes the effects of a large solid angle on the phase-sensitive features of the atomic populations and population inversions with an emphasis on distinguishing between quantum and classical effects. Finally, we end with conclusions in Sec. IX.

\section{PHYSICAL SYSTEM}

The basic structure of the atomic system under consideration is shown on Fig. 1. A three-level atom with eigenstates $|1\rangle,|2\rangle$, and $|3\rangle$ is in a ladder configuration with eigenfrequencies $\left\{\omega_{21}, \omega_{32}, \omega_{31}\right\}$ defined by $\omega_{i j}=\left(E_{i}-E_{j}\right) / \hbar$ and full width at half maximum atomic decay rates given by $\gamma_{2}$ and $\gamma_{3}$ for the transitions $|2\rangle \rightarrow|1\rangle$ and $|3\rangle \rightarrow|2\rangle$, respectively. In addition, we define $\Delta=\left|\left[\left(\omega_{31}\right) / 2\right]-\omega_{32}\right|$ $=\left|\left[\left(\omega_{31}\right) / 2\right]-\omega_{21}\right|$ to characterize the degree of nondegeneracy (i.e., $\Delta$ is the frequency distance of the intermediate excited state 2 from half the two-photon eigenfrequency of the atom; see Fig. 1).

This model atom is assumed to be illuminated by the field with positive frequency component $\hat{\varepsilon}=\hat{\varepsilon}_{0}+\hat{\varepsilon}_{q}$, which is composed of the following two parts: First, the field $\hat{\varepsilon}_{0}$ is

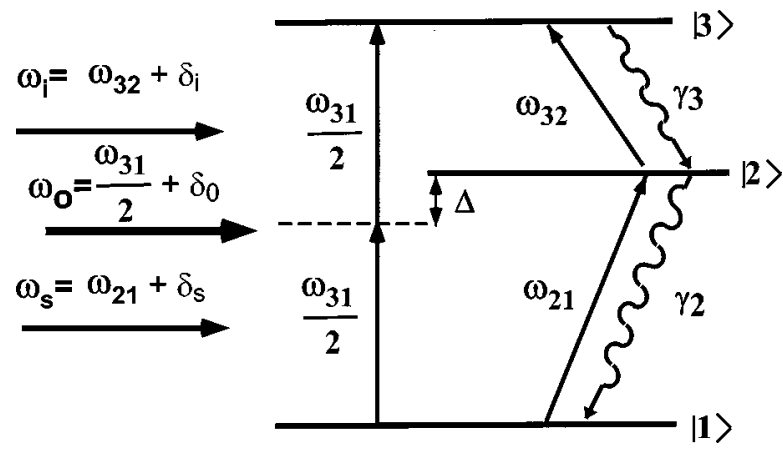

FIG. 1. Schematic representation of the atomic system under consideration.

taken to be in a coherent state with eigenvalue given by $\varepsilon_{0}=\epsilon_{0} e^{-i\left(\omega_{0} t+\phi_{0}\right)}$, where $\epsilon_{0}$ is the amplitude and $\phi_{0}$ the spatial phase of the field at the position of the atom. We will refer to $\varepsilon_{0}$ as the RO. Second, the field $\hat{\varepsilon}_{q}$ is taken to be the output of a subthreshold ND-OPO. As usual [13], the output of the ND-OPO consists of two energy-carrying sidebands, namely, the signal and idler fields with spectral distributions centered at frequencies $\omega_{s, i}=\omega_{0}^{\prime} \pm \Delta^{\prime}$, respectively, and placed symmetrically around the frequency $\omega_{0}^{\prime}$. A real NDOPO can generate many such pairs of sidebands, but, due to atomic resonance conditions described below, only a single pair is relevant for the problem considered here. For the purposes of the present analysis (and as is at the core of our experiment [10]) we assume that the frequency $\omega_{0}$ of the coherent field $\hat{\varepsilon}_{0}$ is the same as that of the central frequency $\omega_{0}^{\prime}$ for the signal and idler fields $\left(\omega_{0}=\omega_{0}^{\prime}\right)$. Therefore, the electromagnetic field illuminating the atoms consists of three main frequency components centered at $\omega_{0}, \omega_{s}=\omega_{0}+\Delta^{\prime}$, and $\omega_{i}=\omega_{0}-\Delta^{\prime}$. The detunings of these components of the driving field from the atomic eigenfrequencies are defined by $\delta_{0}, \delta_{i}$ and $\delta_{s}$ :

$$
\begin{aligned}
& \omega_{0}=\frac{\omega_{31}}{2}+\delta_{0}, \\
& \omega_{s}=\omega_{21}+\delta_{s}, \\
& \omega_{i}=\omega_{32}+\delta_{i} .
\end{aligned}
$$

Since $\omega_{s, i}=\omega_{0} \pm \Delta^{\prime}$, we have that

$$
\begin{aligned}
& \delta_{s}=\delta_{0}+\left(\Delta^{\prime}-\Delta\right), \\
& \delta_{i}=\delta_{0}-\left(\Delta^{\prime}-\Delta\right) .
\end{aligned}
$$

In the subsequent analysis, we assume that $\omega_{0} \approx \omega_{13} / 2$, i.e., $\delta_{0}$ is small, and that $\Delta \approx \Delta^{\prime}$, with the residual detunings $\delta_{s}$ and $\delta_{i}$, on the order of the atomic linewidths $\left(\gamma_{2}, \gamma_{3}\right)$. We also take the quantum field $\hat{\varepsilon}_{q}$ to be a broadband field with respect to the atomic linewidths $\left(\gamma_{2}, \gamma_{3}\right)$, so that $\delta_{s}$ and $\delta_{i}$ do not enter the description of the dynamics of the system. This assumption allows us to avoid the complexity of finite bandwidth squeezed excitation [14]. However, the coherent part of the total excitation field $\hat{\varepsilon}_{0}$ is by definition narrowband, so that the detuning $\delta_{0}$ will be important in the system's dynamics. 
Finally we describe the quantum field $\hat{\varepsilon}_{q}(t)$ in the time domain via the creation and annihilation operators $\hat{a}(\omega)$ and $\hat{a}^{\dagger}(\omega)$ in frequency domain, related to each other as usual by $\hat{\varepsilon}_{q}(t)=\int \sqrt{\left(\hbar \omega^{\prime} / 2 \epsilon_{0} V\right)} \hat{a}\left(\omega^{\prime}\right) e^{i \omega^{\prime} t} d \omega^{\prime}$. The operators $\hat{a}$ and $\hat{a}^{\dagger}$ satisfy the commutation relation

$$
\left[\hat{a}^{\dagger}(\omega), \hat{a}\left(\omega^{\prime}\right)\right]=\delta\left(\omega-\omega^{\prime}\right),
$$

and the nonclassical properties of the quantum field $\hat{\varepsilon}_{q}$ can be summarized in terms of the expectation values of the correlation functions of $\hat{a}$ and $\hat{a}^{\dagger}$ which are taken to be of the following form for the output of a ND-OPO [5]:

$$
\left\langle\hat{a}^{\dagger}(\omega) \hat{a}\left(\omega^{\prime}\right)\right\rangle=N(\omega) \delta\left(\omega-\omega^{\prime}\right)
$$

and

$$
\begin{aligned}
\left\langle\hat{a}(\omega) \hat{a}\left(\omega^{\prime}\right)\right\rangle & =M(\omega) \delta\left(2 \omega_{0}-\omega-\omega^{\prime}\right) \\
& =M\left(2 \omega_{0}-\omega\right) \delta\left(2 \omega_{0}-\omega-\omega^{\prime}\right) .
\end{aligned}
$$

The functions $N(\omega)$ and $M(\omega)$ are slowly varying functions of frequency $\omega$, peaked at the signal and idler frequencies $\omega_{s}$ and $\omega_{i}$, and of width given in terms of the (cold-cavity) linewidth of the ND-OPO. Furthermore, as shown in Ref. [5],

$$
|M(\omega)|^{2} \leqslant N(\omega) N\left(2 \omega_{0}-\omega\right)+\min \left[N(\omega), N\left(2 \omega_{0}-\omega\right)\right],
$$

with the complex number $M$ written as

$$
M(\omega)=|M(\omega)| e^{i \phi_{s}},
$$

where $\phi_{s}$ is the phase of the squeezed vacuum. We define quantum squeezing by the condition $|M(\omega)|^{2}$ $>N(\omega) N\left(2 \omega_{0}-\omega\right)$. Note that for $|M(\omega)|=0$ the statistics of the field become indistinguishable from a thermal source (i.e., a phase-symmetric distribution of fluctuations for the quadrature amplitudes of the field). Also, we will define a "classical" squeezed state to be such that $0<|M(\omega)|^{2}$ $\leqslant N(\omega) N\left(2 \omega_{0}-\omega\right)$ (i.e., an asymmetric phase-space distribution for the quadrature amplitudes, but with a lower bound set by the vacuum fluctuations for the "quiet" quadrature). In Sec. VII, the distinction between the cases of quantum squeezing, classical squeezing, and thermal states will become important when we try to infer the nonclassical statistics of the exciting fields from the properties of the atomic excitation, and hence identify intrinsically quantum effects in the atomic processes.

Before proceeding with the full-theory analysis it is worth recalling the simplified analysis given in Ref. [10], which helps in motivating the subsequent discussion. There, the starting point was Mollow's [15] expression for the twophoton excitation rate, given in terms of the fourth-order correlation function $D(\tau)$ of the excitation field $\hat{\varepsilon}$,

$$
D(\tau) \equiv\left\langle\hat{\varepsilon}^{\dagger}(t+\tau) \hat{\varepsilon}^{\dagger}(t+\tau) \hat{\varepsilon}(t) \hat{\varepsilon}(t)\right\rangle .
$$

Substituting $\hat{\varepsilon}(t)=\hat{\varepsilon}_{0}+\hat{\varepsilon}_{q}$, taking the Fourier transform of $D(\tau)$, and using the relations given by Eqs (4), (5), and (6), leads to

$$
\rho_{33} \propto \Phi(\Omega)=\epsilon_{0}^{4}\left[1+\frac{2 M_{\text {eff }}}{\epsilon_{0}^{2}} \cos \left(2 \phi_{0}+\phi_{s}\right)\right]+2 \epsilon_{0}^{2} N(\Omega),
$$

where

$$
\Phi(\Omega) \equiv(1 / 2 \pi) \int e^{i \Omega \tau} D(\tau) d \tau
$$

and

$$
M_{\mathrm{eff}} \equiv \int\left|M\left(\Omega^{\prime}\right)\right| d \Omega^{\prime} .
$$

From this expression for the excited-state population $\rho_{33}$, it is obvious that $\rho_{33}$ has an oscillating component whose origin is QI. Moreover, by monitoring this oscillating term one obtains a handle on $M_{\text {eff }}$ which is a measure of the nonclassical correlations of the ND-OPO output. However, as explained in Ref. [10], in order to claim that nonclassical observations between the signal and idler outputs of the NDOPO have been observed, one must show that the quantum squeezing condition $|M(\omega)|^{2}>N(\omega) N\left(2 \omega_{0}-\omega\right)$ is satisfied. Unfortunately, the detection of $M_{\text {eff }}$ using QI lacks a direct comparison of $N$ with $M$, although alternative methods have been proposed [10].

For completeness, we note that in the usual homodyne detection of squeezing the Fourier transform of the photocurrent is given by

$$
\begin{aligned}
\Psi(\Omega)= & \epsilon_{0}^{4}\left[1+\frac{2 N_{\text {eff }}}{\epsilon_{0}^{2}}\right]+2 \epsilon_{0}^{2}[N(\Omega) \\
& \left.+|M(\Omega)| \cos \left(2 \phi_{0}+\phi_{s}\right)\right],
\end{aligned}
$$

where $N_{\text {eff }} \equiv \int N\left(\Omega^{\prime}\right) d \Omega^{\prime}$. The second term in this case is proportional to the usual definition of the spectrum of squeezing $[13,16,17]$ given by $S(\Omega, \theta)=2[N(\Omega)+|M(\Omega)| \cos \theta]$, which takes negative values only if the quantum squeezing condition is satisfied.

In summary, comparing the methods of QI and the traditional homodyne detection of squeezing, we emphasize that while QI allows access to nonclassical correlations between fields that differ in frequency by 10 's -100 's of $\mathrm{THz}$ (via $M$ ), the validation of the condition for quantum squeezing is not so trivial. On the other hand while the usual homodyne method automatically compares $M$ with $N$, it cannot detect correlations between fields that are separated in frequency by more than few tens of $\mathrm{GHz}$, because of the technical limitations of the speed of available photodetectors. More details about this issue can be found in Ref. [10].

\section{HAMILTONIAN FORMULATION}

Our system is similar to the one studied by Ficek and Drummond in Ref. [5], with the important addition of the RO field $\hat{\varepsilon}_{0}$. We have therefore chosen the master equation of Ref. [5] as our starting point. Since the bulk of the formalism used here to deal with the quantum fields is drawn from Ref. [5], we will briefly explain the origin of and notations for the various terms. Note, however, that the addition of the classical RO field as part of the total excitation field, 
leads to a rich new phenomenology arising from quantum interference of excitation pathways, which is a new arena within the context of the interaction of squeezed light with atoms.

With this in mind, we begin by writing the total Hamiltonian $H_{\text {tot }}$ of the system as

$$
H_{\mathrm{tot}}=H_{A}+H_{F}+H_{\mathrm{int}},
$$

where $H_{A}$ describes the free evolution of atomic operators, and is given by

$$
H_{A}|m\rangle=E_{m}|m\rangle
$$

for each of the atomic eigenstates $m=1,2$, and 3. $H_{F}$ is the free-field Hamiltonian given by

$$
H_{F}=\hbar \int a^{\dagger}(\omega) a(\omega) \omega d \omega
$$

where the coherent state field $\hat{\varepsilon}_{0}$ has been ignored in $H_{F}$ since it contributes only a $c$ number to the overall energy. The interaction of the field with the atom is described by $H_{\text {int }}$, defined to be

$$
H_{\mathrm{int}}=i \hbar \int d \omega\left\{\left[\sum_{i} \sum_{j \neq i} g_{i j}(\omega) S_{i j}\right] a(\omega)-\text { H.c. }\right\}-i \hbar Q_{o}^{\prime}\left[\left(S_{31} e^{-i 2\left(\delta_{0} t+\phi_{0}\right)}-S_{13} e^{i 2\left(\delta_{0} t+\phi_{0}\right)}\right)+i\left(\beta_{3} S_{33}-\beta_{1} S_{11}\right)\right]
$$

where $g_{i j}$ are coupling coefficients and $S_{i j}$ atomic operators defined by $S_{i j}=|i\rangle\langle j|, i, j=1,2$, and 3 . As usual these operators satisfy the commutation relation

$$
\left[S_{i j}, S_{p q}\right]=S_{i q} \delta_{j p}-S_{p j} \delta_{q i},
$$

where $\delta_{i j}$ is the Kroniger delta function. Note that in the electric-dipole approximation, $g_{13}=0$. The parameter $Q_{o}^{\prime}$ is a "Rabi-like" frequency that takes the form

$$
Q_{o}^{\prime}=\frac{\mu_{1} \mu_{2} \epsilon_{0}^{2}}{4 \Delta \hbar^{2}}
$$

where $\mu_{1}$ and $\mu_{2}$ are the dipole moments that couple the $|1\rangle \leftrightarrow|2\rangle$ and $|2\rangle \leftrightarrow|3\rangle$ transitions, respectively.

Before proceeding further let us take a moment to examine Eq. (15) beginning with the first term which describes the interaction of the atoms with the quantum field $\hat{\varepsilon}_{q}$ and which has been extensively discussed in Ref. [5]. This first term is responsible for driving the $|1\rangle \leftrightarrow|2\rangle$ and $|2\rangle \leftrightarrow|3\rangle$ atomic transitions. To account for the additional coherent-state component of the field $\hat{\varepsilon}_{0}$, we add to the interaction Hamiltonian the second term of Eq. (15), which is an effective Hamiltonian of the form analogous to that for the semiclassical interaction of a coherent field with an atomic dipole [18]. However, because this term describes a two-photon transi- tion, the usual Rabi frequency $\Omega \propto \epsilon r$ has been replaced with the two-photon analog given by $Q_{o}^{\prime} \propto \epsilon^{2} r^{2}$ (note that, for a three-level system, $\langle r\rangle_{12}\langle r\rangle_{23}=\left\langle r^{2}\right\rangle_{13}$ ).

This form of an effective Hamiltonian has been extensively used for the study of two-photon absorption by many authors [19-22], and is valid under the assumption that the three-level atom is far from degenerate [23] (i.e., $\omega_{i j} \gg \Delta$ $\gg \delta_{k}$ ), which we assume to be the case in our work as well. The parameters $\beta_{1}$ and $\beta_{3}$ quantify the strength of the intensity-dependent Stark shifts of the $|1\rangle$ and $|3\rangle$ levels, respectively, due to the virtual transition to the intermediate (|2〉) level [22], and can be shown from Ref. [20] [Eq. (63)] and the definition of $Q_{o}^{\prime}$ to be related to each other by

$$
\beta_{1}=\frac{1}{\beta_{3}}=\frac{\left|\mu_{2}\right|}{\left|\mu_{1}\right|} \simeq\left(\frac{\gamma_{3}}{\gamma_{2}}\right)^{1 / 2}
$$

where the last equality follows from Eq. (21) in Ref. [5].

\section{MASTER EQUATION}

To simplify the subsequent analysis, we rewrite the total Hamiltonian $H_{\text {tot }}$ as being composed of two parts, namely,

$$
H_{\mathrm{tot}}=H_{\mathrm{FD}}+H_{0},
$$

where $H_{\mathrm{FD}}$ is the part of the Hamiltonian analyzed by Ficek and Drummond in Ref. [5] given by

$$
H_{\mathrm{FD}}=H_{A}+H_{F}+i \hbar \int d \omega\left\{\left[\sum_{i} \sum_{j \neq i} g_{i j}(\omega) S_{i j}\right] a(\omega)-\text { H.c. }\right\},
$$

while $H_{0}$ is an "effective" interaction Hamiltonian given by

$$
H_{0}=-i \hbar Q_{o}^{\prime}\left[\left(S_{31} e^{-2 i\left(\delta_{0} t+\phi_{0}\right)}-S_{13} e^{2 i\left(\delta_{0} t+\phi_{0}\right)}\right)+i\left(\beta_{3} S_{33}-\beta_{1} S_{11}\right)\right],
$$

and describes the addition of the RO driving field $\hat{\varepsilon}_{0}$.

From Eq. (19), we derive a master equation for the atom, composed of two parts, 


$$
\frac{\partial \rho}{\partial t}=\left(\frac{\partial \rho}{\partial t}\right)_{\mathrm{FD}}-Q_{o}^{\prime}\left[\left(S_{31} e^{-2 i\left(\delta_{0} t+\phi_{0}\right)}-S_{13} e^{2 i\left(\delta_{0} t+\phi_{0}\right)}\right)-i\left(\beta_{3} S_{33}-\beta_{1} S_{11}\right), \rho\right],
$$

with the first term $(\partial \rho / \partial t)_{\mathrm{FD}}$ being identical to that analyzed by Ficek and Drummond in Ref. [5], and the second describing the interaction of the atom with the additional $\hat{\varepsilon}_{0}$ field. Furthermore, we adopt the simplified notation of Ref. [5], namely, $S_{i}^{-}=\left(S_{i}^{+}\right)^{\dagger} \equiv S_{i, i+1}$ and $\omega_{i} \equiv \omega_{i+1, i}$. Then, expanding Eq. (22) results in the following expression:

$$
\begin{aligned}
\frac{\partial \rho}{\partial t}= & -\sum_{i, j=1}^{2}\left[M\left(\omega_{i}\right) \eta_{i j}-i \Delta \omega_{i j}\right]\left(S_{i}^{+} \rho S_{j}^{+}-S_{j}^{+} S_{i}^{+} \rho+S_{j}^{+} \rho S_{i}^{+}-\rho S_{i}^{+} S_{j}^{+}\right) e^{i\left(\omega_{i}+\omega_{j}-2 \omega_{o}\right) t}-\sum_{i, j=1}^{2}\left[M^{*}\left(\omega_{i}\right) \eta_{i j}^{*}+i \Delta \omega_{i j}^{*}\right] \\
& \times\left(S_{i}^{-} \rho S_{j}^{-}-S_{j}^{-} S_{i}^{-} \rho+S_{j}^{-} \rho S_{i}^{-}-\rho S_{i}^{-} S_{j}^{-}\right) e^{-i\left(\omega_{i}+\omega_{j}-2 \omega_{o}\right) t}-\sum_{i, j=1}^{2} N\left(\omega_{i}\right) \gamma_{i j}\left(\rho S_{j}^{-} S_{i}^{+}+S_{j}^{-} S_{i}^{+} \rho-2 S_{i}^{+} \rho S_{j}^{-}\right) e^{i\left(\omega_{i}-\omega_{j}\right) t} \\
& -\sum_{i, j=1}^{2}\left[N\left(\omega_{i}\right)+1\right] \gamma_{i j}\left(\rho S_{i}^{+} S_{j}^{-}+S_{i}^{+} S_{j}^{-} \rho-2 S_{j}^{-} \rho S_{i}^{+}\right) e^{i\left(\omega_{i}-\omega_{j}\right) t}-i \sum_{i=1}^{2}\left(\Delta \omega_{i}^{0}+\Delta \omega_{i}\right)(|i\rangle\langle i|\rho-\rho| i\rangle\langle i|) \\
& -Q_{o}^{\prime}\left[\left(S_{31} \rho-\rho S_{31}\right) e^{-2 i\left(\delta_{0} t+\phi_{0}\right)}-\left(S_{13} \rho-\rho S_{13}\right) e^{2 i\left(\delta_{0} t+\phi_{0}\right)}\right]+i Q_{o}^{\prime}\left[\beta_{3}\left(S_{33} \rho-\rho S_{33}\right)-\beta_{1}\left(S_{11} \rho-\rho S_{11}\right)\right]
\end{aligned}
$$

where the first five terms correspond to the results of Ficek and Drummond of Ref. [5(a)], Eq. (20), while the last two terms are due to $\hat{\varepsilon}_{0}$. In the above equation $\gamma_{i i}, i=1$ and 2 , are equal to half the radiative decay constants for the $|1\rangle \rightarrow|2\rangle$ and $|2\rangle \rightarrow|3\rangle$ transitions (hence $\gamma_{11}=\gamma_{2} / 2$ and $\left.\gamma_{22}=\gamma_{3} / 2\right)$. The additional damping terms $\gamma_{12}$ and $\gamma_{21}$ [as defined in Eq. (21) of Ref. [5(a)]] are in general nonzero, but because we assume that the atom has nonequidistant energy levels, with $\Delta$ large, these terms are rapidly oscillating and may be dropped. In the above equation, the presence of squeezing and the fact that $M=\langle a a\rangle \neq 0$ introduce the additional damping constants $\eta_{i j}$ [as defined in Eq. (21) of Ref. [5(a)]] which are similar to the more traditional $\gamma_{i j}$. However, relative to $\gamma_{i j}$, the situation is reversed for $\eta_{i j}$, for which only $\eta_{12}$ and $\eta_{21}$ contribute, while the terms $\eta_{11}$ and $\eta_{22}$ enter in fast oscillating terms that may be neglected. In addition, it can be shown from Ref. [5] that $\eta_{12}=\eta_{21}^{*}$ $=\frac{1}{2} \sqrt{\gamma_{2} \gamma_{3}}$, where any nonzero phase associated with $\eta_{12}$ has been absorbed without loss of generality into the phase of the complex number $M$. The terms $\Delta \omega_{i j}$ are frequency shifts caused by the presence of squeezing, while $\Delta \omega_{i}$ and $\Delta \omega_{i}^{0}$ are the more familiar Stark and Lamb shifts, respectively [explicitly defined in Eq. (21) of Ref. [5(a)]]. More details and explicit derivation formulas for all the parameters $\eta_{i j}, \gamma_{i j}$, $\Delta \omega_{i j}, \Delta \omega_{i}$, and $\Delta \omega_{i}^{0}$ can be found in Ref. [5].

\section{EQUATIONS OF MOTION}

Next, we expand Eq. (23) for the various components of the density matrix, which leads to the following differential equations for the atomic populations $\rho_{11}, \rho_{22}$, and $\rho_{33}$ :

$$
\begin{aligned}
\frac{\partial \rho_{11}}{\partial t}= & \left(M\left(\omega_{2}\right) \eta_{21}-i \Delta \omega_{21}\right) \rho_{13} e^{-2 i \delta_{0} t}+\left(M^{*}\left(\omega_{2}\right) \eta_{21}^{*}\right. \\
& \left.+i \Delta \omega_{21}^{*}\right) \rho_{31} e^{2 i \delta_{0} t}-N\left(\omega_{1}\right) \gamma_{2} \rho_{11}+\left(N\left(\omega_{1}\right)+1\right) \gamma_{2} \rho_{22} \\
& +Q_{o}^{\prime}\left(\rho_{13} e^{-2 i\left(\delta_{0} t+\phi_{0}\right)}+\rho_{31} e^{2 i\left(\delta_{0} t+\phi_{0}\right)}\right)
\end{aligned}
$$

$$
\begin{aligned}
\frac{\partial \rho_{22}}{\partial t}= & -\left\{\left[M\left(\omega_{1}\right) \eta_{12}-i \Delta \omega_{12}\right]+\left[M\left(\omega_{2}\right) \eta_{21}\right.\right. \\
& \left.\left.-i \Delta \omega_{21}\right]\right\} \rho_{13} e^{-2 i \delta_{0} t}-\left\{\left[M^{*}\left(\omega_{2}\right) \eta_{21}^{*}+i \Delta \omega_{21}^{*}\right]\right. \\
& \left.+\left[M^{*}\left(\omega_{1}\right) \eta_{12}^{*}+i \Delta \omega_{12}^{*}\right]\right\} \rho_{31} e^{2 i \delta_{0} t}-N\left(\omega_{2}\right) \gamma_{3} \rho_{22} \\
& +N\left(\omega_{1}\right) \gamma_{2} \rho_{11}-\left(N\left(\omega_{1}\right)+1\right) \gamma_{2} \rho_{22} \\
& +\left[N\left(\omega_{2}\right)+1\right] \gamma_{3} \rho_{33},
\end{aligned}
$$

and

$$
\begin{aligned}
\frac{\partial \rho_{33}}{\partial t}= & {\left[M\left(\omega_{1}\right) \eta_{12}-i \Delta \omega_{12}\right] \rho_{13} e^{-2 i \delta_{0} t}+\left[M^{*}\left(\omega_{1}\right) \eta_{12}^{*}\right.} \\
& \left.+i \Delta \omega_{12}^{*}\right] \rho_{31} e^{2 i \delta_{0} t}+N\left(\omega_{2}\right) \gamma_{3} \rho_{22}-\left(N\left(\omega_{2}\right)+1\right) \gamma_{3} \rho_{33} \\
& -Q_{o}^{\prime}\left(\rho_{13} e^{-2 i\left(\delta_{0} t+\phi_{0}\right)}+\rho_{31} e^{2 i\left(\delta_{0} t+\phi_{0}\right)}\right)
\end{aligned}
$$

while for the atomic coherences $\rho_{12}, \rho_{23}$, and $\rho_{13}$ we obtain

$$
\begin{aligned}
\frac{\partial \rho_{12}}{\partial t}= & \left(M^{*}\left(\omega_{2}\right) \eta_{21}^{*}+i \Delta \omega_{21}^{*}\right) \rho_{32} e^{2 i \delta_{0} t}-\frac{1}{2}\left[N\left(\omega_{2}\right) \gamma_{3}\right. \\
& \left.+N\left(\omega_{1}\right) \gamma_{2}+\left(N\left(\omega_{1}\right)+1\right) \gamma_{2}\right] \rho_{12}+i\left[\left(\Delta \omega_{2}^{0}+\Delta \omega_{2}\right)\right. \\
& \left.-\left(\Delta \omega_{1}^{0}+\Delta \omega_{1}\right)\right] \rho_{12}+Q_{o}^{\prime} \rho_{32} e^{2 i\left(\delta_{0} t+\phi_{0}\right)}-i \beta_{1} Q_{o}^{\prime} \rho_{12},
\end{aligned}
$$

$$
\begin{aligned}
\frac{\partial \rho_{23}}{\partial t}= & \left(M^{*}\left(\omega_{1}\right) \eta_{12}^{*}+i \Delta \omega_{12}^{*}\right) \rho_{21} e^{2 i \delta_{0} t}-\frac{1}{2}\left\{N\left(\omega_{2}\right) \gamma_{3}\right. \\
& \left.+\left[N\left(\omega_{2}\right)+1\right] \gamma_{3}+\left[N\left(\omega_{1}\right)+1\right] \gamma_{2}\right\} \rho_{23} \\
& -i\left(\Delta \omega_{2}^{0}+\Delta \omega_{2}\right) \rho_{23}-Q_{o}^{\prime} \rho_{21} e^{2 i\left(\delta_{0} t+\phi_{0}\right)} \\
& -i \beta_{3} Q_{o}^{\prime} \rho_{23}
\end{aligned}
$$

and 


$$
\begin{aligned}
\frac{\partial \rho_{13}}{\partial t}= & -\left\{\left[M^{*}\left(\omega_{1}\right) \eta_{12}^{*}+i \Delta \omega_{12}^{*}\right]+\left[M^{*}\left(\omega_{2}\right) \eta_{21}^{*}\right.\right. \\
& \left.\left.+i \Delta \omega_{21}^{*}\right]\right\} \rho_{22} e^{2 i \delta_{0} t}+\left[M^{*}\left(\omega_{2}\right) \eta_{21}^{*}+i \Delta \omega_{21}^{*}\right] \rho_{33} e^{2 i \delta_{0} t} \\
& +\left[M^{*}\left(\omega_{1}\right) \eta_{12}^{*}+i \Delta \omega_{12}^{*}\right] \rho_{11} e^{2 i \delta_{0} t}-\frac{1}{2}\left[N\left(\omega_{1}\right) \gamma_{2}\right. \\
& \left.+\left(N\left(\omega_{2}\right)+1\right) \gamma_{3}\right] \rho_{13}-i\left(\Delta \omega_{1}^{0}+\Delta \omega_{1}\right) \rho_{13}+Q_{o}^{\prime} \\
& \times\left(\rho_{33}-\rho_{11}\right) e^{2 i\left(\delta_{0} t+\phi_{0}\right)}-i\left(\beta_{1}+\beta_{3}\right) Q_{o}^{\prime} \rho_{13} .
\end{aligned}
$$

Finally, the last three components of the density matrix, $\rho_{21}$, $\rho_{32}$, and $\rho_{31}$ are given by the complex conjugates of Eqs. (27)-(29) since $\rho_{i j}=\rho_{j i}^{*}$. Also note that due to the trace condition $\operatorname{Tr}(\rho)=1$, only eight out of the nine components of the density matrix $\rho_{i j}, i, j \in\{1,2,3\}$, are independent, which implies that the differential equations (24)-(26) are not independent, but satisfy $\left(\partial \rho_{11} / \partial t\right)+\left(\partial \rho_{22} / \partial t\right)+\left(\partial \rho_{33} / \partial t\right)$ $=0$. Note that these equations reduce to Eq. (3) of Ref. [5(b)] if the two-photon excitation $Q_{o}^{\prime}=0$.

At this point, we will deviate from Ficek and Drummond's notation and introduce a normalization that will cast all our equations into a dimensionless form. In particular we define a new dimensionless time $\tau$ to be

$$
\tau=\gamma t,
$$

where $\gamma \equiv \sqrt{\gamma_{2} \gamma_{3}}$. In addition all frequencies and detunings of the problem will be normalized to $\gamma$, beginning with the following normalized quantities:

$$
\begin{gathered}
\delta_{i j} \equiv \frac{\Delta \omega_{i j}}{\gamma}, \\
\delta_{i}^{S} \equiv \frac{\Delta \omega_{i}}{\gamma}, \\
\delta_{i}^{L} \equiv \frac{\Delta \omega_{i}^{0}}{\gamma},
\end{gathered}
$$

where $\delta_{i j}$ are the normalized frequency shifts due to the squeezing, $\delta_{i}^{S}$ are the normalized Stark shifts, and $\delta_{i}^{L}$ are the normalized Lamb shifts. Similarly, we normalize the twophoton Rabi frequency to $Q_{o} \equiv Q_{o}^{\prime} / \gamma$ and the RO detuning from the two-photon transition to $\delta_{0} \rightarrow \delta_{0} / \gamma$, where, for notational simplicity, $\delta_{0}$ will now imply a normalized quantity. By making these substitutions and change of variables in Eqs. (24)-(29) we arrive at the following set of coupled differential equations for the independent elements of the density matrix:

$$
\begin{gathered}
\frac{\partial \rho_{22}}{\partial \tau}=-\left[M-i\left(\delta_{12}+\delta_{21}\right)\right] \rho_{13} e^{-2 i \delta_{0} \tau}-\left[M^{*}+i\left(\delta_{21}^{*}+\delta_{12}^{*}\right)\right] \rho_{31} e^{2 i \delta_{0} \tau}+\alpha N_{1}\left(1-\rho_{22}-\rho_{33}\right)-\alpha\left(N_{1}+1\right) \rho_{22}-\frac{1}{\alpha} N_{2} \rho_{22} \\
+\frac{1}{\alpha}\left(N_{2}+1\right) \rho_{33}, \\
\frac{\partial \rho_{33}}{\partial \tau}=\left(\frac{1}{2} M-i \delta_{12}\right) \rho_{13} e^{-2 i \delta_{0} \tau}+\left(\frac{1}{2} M^{*}+i \delta_{12}^{*}\right) \rho_{31} e^{2 i \delta_{0} \tau}+\frac{1}{\alpha} N_{2} \rho_{22}-\frac{1}{\alpha}\left(N_{2}+1\right) \rho_{33}-Q_{o}\left[\rho_{13} e^{-2 i\left(\delta_{0} \tau+\phi_{0}\right)}+\rho_{31} e^{2 i\left(\delta_{0} \tau+\phi_{0}\right)}\right] \\
\quad \begin{aligned}
\frac{\partial \rho_{12}}{\partial \tau}=\left(\frac{1}{2} M^{*}+i \delta_{12}^{*}\right) \rho_{32} e^{2 i \delta_{0} \tau}-\frac{1}{2}\left(N_{2} \frac{1}{\alpha}+N_{1} \alpha+\left(N_{1}+1\right) \alpha\right) \rho_{12}+i\left[\left(\delta_{2}^{L}+\delta_{2}^{S}\right)-\left(\delta_{1}^{L}+\delta_{1}^{S}\right)-\frac{1}{\alpha} Q_{o}\right] \rho_{12} \\
\quad+Q_{o} \rho_{32} e^{2 i\left(\delta_{0} \tau+\phi_{0}\right)}, \\
\frac{\partial \rho_{23}}{\partial \tau}=\left(\frac{1}{2} M^{*}+i \delta_{12}^{*}\right) \rho_{21} e^{2 i \delta_{0} \tau}-\frac{1}{2}\left[N_{2} \frac{1}{\alpha}+\left(N_{2}+1\right) \frac{1}{\alpha}+\left(N_{1}+1\right) \alpha\right] \rho_{23}-i\left[\left(\delta_{2}^{L}+\delta_{2}^{S}\right)+\alpha Q_{o}\right] \rho_{23}-Q_{o} \rho_{21} e^{2 i\left(\delta_{0} \tau+\phi_{0}\right)}
\end{aligned}
\end{gathered}
$$

and

$$
\begin{aligned}
\frac{\partial \rho_{13}}{\partial \tau}= & -\left[M^{*}+i\left(\delta_{21}^{*}+\delta_{12}^{*}\right)\right] \rho_{22} e^{2 i \delta_{0} \tau}+i\left(\delta_{21}^{*}-\delta_{12}^{*}\right) \rho_{33} e^{2 i \delta_{0} \tau}+\left(\frac{1}{2} M^{*}+i \delta_{12}^{*}\right)\left(1-\rho_{22}\right) e^{2 i \delta_{0} \tau}-\frac{1}{2}\left[N_{1} \alpha+\left(N_{2}+1\right) \frac{1}{\alpha}\right] \rho_{13} \\
& -i\left[\left(\delta_{1}^{L}+\delta_{1}^{S}\right)+\left(\alpha+\frac{1}{\alpha}\right) Q_{o}\right] \rho_{13}+Q_{o}\left(2 \rho_{33}+\rho_{22}-1\right) e^{2 i\left(\delta_{0} \tau+\phi_{0}\right)}
\end{aligned}
$$


In Eqs. (34)-(38), we used the simplified notation $N_{i}=N\left(\omega_{i}\right)$ and $M=M\left(\omega_{i}\right)$ for $i=1$ and 2, defined $\alpha$ to be

$$
\alpha \equiv\left(\frac{\gamma_{2}}{\gamma_{3}}\right)^{1 / 2}
$$

and made the substitution $\rho_{11}=1-\rho_{22}-\rho_{33}$.

\section{ATOMIC POPULATIONS}

While the above set of coupled differential equations (34)-(38) together with the complex conjugates of Eqs. (36)-(38) fully describes the dynamics of the atomic system in terms of its density matrix, here we are only interested in the atomic populations. It is easy to see that the derivation of the populations is simplified by the fact that the equations of motion decouple into two groups of linearly independent differential equations. In particular, to solve for the atomic populations we only need to consider the closed set of differential equations for $\rho_{22}, \rho_{33}, \rho_{13}$, and $\rho_{31}$. By also neglecting frequency shifts due to the quantum field $\hat{\varepsilon}_{q}\left(\delta^{L}\right.$ and $\delta^{S}$ ) which are small corrections as discussed in Ref. [5], we solve for the steady-state cases $\partial \rho_{22} / \partial \tau=0, \partial \rho_{33} / \partial \tau=0$, $\partial\left(\rho_{13} e^{-2 i \delta_{0} \tau}\right) / \partial \tau=0$, and $\partial\left(\rho_{31} e^{2 i \delta_{0} \tau}\right) / \partial \tau=0$, and find a simple algebraic set of equations to describe the population dynamics of the atom. These equations are

$$
\begin{gathered}
M \tilde{\rho}_{13}+M^{*} \tilde{\rho}_{13}^{*}=\alpha N_{1}-\left(\alpha+2 \alpha N_{1}+\frac{1}{\alpha} N_{2}\right) \rho_{22} \\
+\left(\frac{1}{\alpha}-\alpha N_{1}+\frac{1}{\alpha} N_{2}\right) \rho_{33}, \\
\left(Q_{o} e^{\left.-2 i \phi_{0}-\frac{1}{2} M\right) \tilde{\rho}_{13}+\left(Q_{o} e^{2 i \phi_{0}}-\frac{1}{2} M^{*}\right) \tilde{\rho}_{13}^{*}}\right. \\
=\frac{1}{\alpha} N_{2} \rho_{22}-\frac{1}{\alpha}\left(N_{2}+1\right) \rho_{33},
\end{gathered}
$$

$$
\begin{aligned}
\tilde{\rho}_{13}= & \frac{1}{A}\left(-\frac{3}{2} M^{*}+Q_{o} e^{2 i \phi_{0}}\right) \rho_{22}+2 \frac{1}{A} Q_{o} e^{2 i \phi_{0}} \rho_{33} \\
& +\frac{1}{A}\left(\frac{1}{2} M^{*}-Q_{o} e^{2 i \phi_{0}}\right)
\end{aligned}
$$

where $\tilde{\rho}_{13}$ is defined to be

$$
\tilde{\rho}_{13} \equiv \rho_{13} e^{-2 i \delta_{0} \tau}
$$

and the quantity $A$ is given by

$$
A \equiv \frac{1}{2}\left[N_{1} \alpha+\left(N_{2}+1\right) \frac{1}{\alpha}\right]+i\left[2 \delta_{0}+\left(\alpha+\frac{1}{\alpha}\right) Q_{o}\right]
$$

As we can see, the detuning of the RO field from the twophoton resonance, $\delta_{0}=\omega_{0}-\left(\omega_{13} / 2\right)$, is the only detuning that enters the solution, as we indicated in Sec. II. Finally, substituting Eq. (42) and its complex conjugate into Eqs. (40) and (41) results in a set of two equations for $\rho_{22}$ and $\rho_{33}$, which after some algebra can be written in the following forms:

$$
\Lambda_{1} \rho_{22}+\Xi_{1} \rho_{33}=1
$$

and

$$
\Lambda_{2} \rho_{22}+\Xi_{2} \rho_{33}=1
$$

For the special case when the coherent-field $\hat{\varepsilon}_{0}$ is resonant with the two-photon transition (i.e., for $\delta_{0}=0$ ), and assuming that $\hat{\varepsilon}_{0}$ is a weak field so that the Stark shift contribution (i.e., $\left.[\alpha+(1 / \alpha)] Q_{o}\right)$ can be neglected, the quantities $\Lambda_{1}$, $\Xi_{1}, \Lambda_{2}$, and $\Xi_{2}$ can be shown to be equal to

$$
\begin{gathered}
\Lambda_{1}=\frac{3|M|^{2}-2 Q_{o}|M| \cos \Phi-\frac{1}{2}\left[N_{1} \alpha+\left(N_{2}+1\right) \frac{1}{\alpha}\right]\left(\alpha+2 \alpha N_{1}+\frac{1}{\alpha} N_{2}\right)}{|M|^{2}-2|M| Q_{o} \cos \Phi-\frac{1}{2} \alpha N_{1}\left[N_{1} \alpha+\left(N_{2}+1\right) \frac{1}{\alpha}\right]}, \\
\Xi_{1}=\frac{-4 Q_{o}|M| \cos \Phi+\frac{1}{2}\left[N_{1} \alpha+\left(N_{2}+1\right) \frac{1}{\alpha}\right]\left(\frac{1}{\alpha}-\alpha N_{1}+\frac{1}{\alpha} N_{2}\right)}{|M|^{2}-2|M| Q_{o} \cos \Phi-\frac{1}{2} \alpha N_{1}\left[N_{1} \alpha+\left(N_{2}+1\right) \frac{1}{\alpha}\right]}, \\
\Lambda_{2}=\frac{4 Q_{o}^{2}+3|M|^{2}-8 Q_{o}|M| \cos \Phi-\frac{1}{\alpha}\left[N_{1} \alpha+\left(N_{2}+1\right) \frac{1}{\alpha}\right] N_{2}}{4 Q_{o}^{2}+|M|^{2}-4 Q_{o}|M| \cos \Phi},
\end{gathered}
$$

and

$$
\Xi_{2}=\frac{8 Q_{o}^{2}-4 Q_{o}|M| \cos \Phi+\frac{1}{\alpha}\left[N_{1} \alpha+\left(N_{2}+1\right) \frac{1}{\alpha}\right]\left(N_{2}+1\right)}{4 Q_{o}^{2}+|M|^{2}-4 Q_{o}|M| \cos \Phi},
$$


while in the more general case where $\delta_{0} \neq 0$ these expressions become much more complicated. In Eqs. (47)-(50) the phase $\Phi$ is a combination of the phases of the squeezing $\phi_{s}$ and of the RO $\phi_{0}$, and is given by

$$
\Phi=2 \phi_{0}+\phi_{s} .
$$

Hence the solutions for the atomic populations $\rho_{22}$ and $\rho_{33}$ are given by

$$
\rho_{22}=\frac{\Xi_{1}-\Xi_{2}}{\Lambda_{2} \Xi_{1}-\Xi_{2} \Lambda_{1}}
$$

and

$$
\rho_{33}=\frac{\Lambda_{2}-\Lambda_{1}}{\Lambda_{2} \Xi_{1}-\Xi_{2} \Lambda_{1}} .
$$

In the subsequent discussion we concentrate on this special case for which $\delta_{0}=0$ and for which the above solutions are valid with parameters given by Eqs. (47)-(50).

Note that from the above solution it is clear that the atomic populations have a phase-sensitive modulation determined through the dependence of $\Lambda_{i}$ and $\Xi_{i}$ on $\Phi$, which is the only phase left in the final answer. Furthermore, we see that all phase-sensitive terms are of the form $|M| Q_{o} \cos \Phi$, corresponding to interference between the RO field as described by $Q_{o}$ and the quantum field output of the ND-OPO as described by the correlations of the field-quadrature fluctuations given by $M$. However, since no cross (interference) terms between $N_{1}, N_{2}$, and $Q_{o}$ exist, we conclude that these correlation functions of the fields must be incoherent with each other. Indeed, as shown in Ref. [17], the signal and idler outputs of the ND-OPO, when viewed in isolation from each other, are described by their total intensities $N_{1}$ and $N_{2}$, respectively, and correspond to incoherent thermal fields and hence should not be expected to interfere with $Q_{o}$, although they do contribute to the overall excitation probability by virtue of the energy they carry. More explicitly, note that for excitation with thermal fields $(M=0)$ the coupling between $\rho_{33}$ and $\rho_{13}$, due to the signal and idler fields, vanishes. By contrast, the quantum correlations between the signal and idler fields as given by $M$ drive a nonzero coupling of $\rho_{13}$ with $\rho_{33}$, as does $Q_{o}$, (assuming the RO and squeezed fields are phase coherent), thus giving rise to QI.

Concluding this section, we note that the precise way in which $\Phi$ enters the populations is not completely obvious from Eqs. (52) and (53), especially in view of the five additional parameters, $\left(M, N_{1}, N_{2}, Q_{o}\right.$, and $\left.\alpha\right)$ and the algebraic complexity of the solution. As a result, a global presentation of all the features of our solution is not possible here, so that we will instead focus on a few cases of special interest.

\section{SQUEEZING OVER A SMALL SOLID ANGLE}

\section{Phase-sensitive populations and quantum optical effects}

In the discussion so far we have implicitly assumed that the squeezing illuminates the atom from the full $4 \pi$ solid angle. However in a typical experimental setup, as for example in Refs. [8] and [10], the squeezed field will be focused onto the atoms only over a small angle. In that case, as discussed in Ref. [5], the parameters $N_{1}, N_{2}$, and $M$ should be replaced by $N_{1} v(\theta), N_{2} v(\theta)$, and $M v(\theta)$, where the function $v(\theta)$ is given by

$$
v(\theta)=\frac{1}{2}\left[1-\frac{1}{4}\left(3+\cos ^{2} \theta\right) \cos \theta\right], \quad \theta \in(0, \pi),
$$

with $\theta$ the angle over which squeezing is propagated. For reasonable experimental parameters with focusing angles, $\theta \lesssim 20^{\circ}$, the values that $v(\theta)$ takes are also small, $v(\theta) \leqq 0.04$, justifying a power expansion of the solutions given by Eqs. (52) and (53) with respect to the variable $v(\theta)$, which after some algebra reduce to

$$
\rho_{22} \approx N_{1} v(\theta)+O\left[v^{2}(\theta)\right]
$$

and

$$
\begin{aligned}
\rho^{33} \approx & {\left[4 \alpha^{2} \Omega_{o}^{2}+\left(|M|^{2} \alpha^{2}+N_{1} N_{2}\right) v^{2}(\theta)\right.} \\
& \left.-4 \alpha^{2} Q_{o}|M| v(\theta) \cos \Phi\right]+O\left[v^{3}(\theta)\right] .
\end{aligned}
$$

From Eq. (55), we see that the excited-state population $\rho_{22}$ of the $|2\rangle$ level, to the lowest term in $v(\theta)$, is proportional to the power of the resonant to the $|1\rangle \rightarrow|2\rangle$ transition field component, as given by the field's occupation number $N_{1}$. We also observe that $\rho_{22}$ exhibits no signs of modulation to this order of $v(\theta)$.

However, the population $\rho_{33}$ of the $|3\rangle$ excited state [Eq. (56)] has a more complex behavior, with separate contributions from the coherent reference oscillator field described by $Q_{o}$ which is responsible for the direct $1 \rightarrow 3$ transition [the first term in Eq. (56)]; the quantum field described by $N_{1}$, $N_{2}$, and $M$ which is responsible for the stepwise excitation $1 \rightarrow 2 \rightarrow 3$ [the second term in Eq. (56)]; and finally a cross term due to quantum interference of the amplitudes of these two processes [the third term in Eq. (56)]. However, inspection of this quantum interference term shows that the interference occurs between the amplitude for excitation by the RO field, and only part of the total amplitude for excitation due to the squeezed fields. In particular, if we define $X_{o} \equiv 2 \alpha Q_{o}$ and $X_{q} \equiv \sqrt{X_{q 1}^{2}+X_{q 2}^{2}}$ [with $X_{q 1} \equiv \alpha|M| v(\theta)$ and $\left.X_{q 2} \equiv \sqrt{N_{1} N_{2}} v(\theta)\right]$ to be the excitation amplitudes due to the coherent field $\hat{\varepsilon}_{0}$ and the quantum field $\hat{\varepsilon}_{q}$, respectively, we see that the excited-state population is given by

$$
\rho_{33} \approx\left(X_{0}^{2}-2 X_{0} X_{q 1} \cos \Phi+X_{q 1}^{2}\right)+X_{q 2}^{2} .
$$

Written in this form, $\rho_{33}$ is obviously the result of two quantum interfering pathways with amplitudes $X_{o}$ and $X_{q 1}$, plus a third incoherent contribution whose amplitude is $X_{q 2}$. The physical interpretation of this observation is that excitation via the RO and $M$ interfere via the $\rho_{13}$ coherence, where excitation via $N_{1}$ and $N_{2}$ proceeds incoherently as discussed in Sec. VI, so that their contribution to the total excitation probability adds incoherently.

Finally, we point out that an experimentally relevant limit is that of a strong $\mathrm{RO}$ field relative to the quantum field $\left(\left|X_{0}\right| \gg\left|X_{q}\right|\right)$, in which case the second term in Eq. (56) becomes negligibly small compared to the other two, and hence the result for $\rho_{33}$ simplifies to 


$$
\rho_{33} \approx 4 \alpha^{2} Q_{o}^{2}\left[1-\frac{|M|}{Q_{o}} v(\theta) \cos \Phi\right] .
$$

From this final expression, we see that by measuring the modulation of the excited-state population, the quantity $M$ can be inferred, and hence an access is gained to the nonclassical statistics of the squeezed field as described by the correlation function $M(\Omega)$, where, from Eq. (7), $\left\langle a(\Omega) a\left(\Omega^{\prime}\right)\right\rangle=M(\Omega) \delta\left(\Omega+\Omega^{\prime}\right)$, with $\Omega$ and $\Omega^{\prime}$ defined as the offset frequencies for the signal and idler fields in a rotating frame at $\omega_{0}$ (i.e., $\omega_{s}=\omega_{0}+\Omega$ and $\omega_{i}=\omega_{0}-\Omega^{\prime}$ ). Note that the signal and idler fields can have substantial frequency offsets, so that $\Omega / \omega_{o}$ is not necessarily a small quantity (in the particular example studied in Ref. [10], $\mid \omega_{s}$ $-\omega_{i} \mid / 2 \pi \simeq 25 \mathrm{THz}$ ). This large frequency offset will nonetheless be "demodulated" by the atom which is acting as a nonlinear mixer [12]. We suggest that this capability opens new avenues for the detection of nonclassical correlations at ultrahigh frequency offsets which are beyond the range of conventional homodyne detection, and which we previously discussed in Ref. [10]. In particular, note that the form of quadrature amplitudes change from the usual case in quantum optics for which $\Omega / \omega_{0} \ll 1 \quad[24,25]$.

From an experimental point of view, it is desirable to maximize the signal-to-noise ratio of the observation, which in the context of Eq. (58) implies that we would like to maximize $4 \alpha^{2} Q_{o}^{2}$, and simultaneously have that $|M| v(\theta)$ $\approx Q_{o}$. This combination guarantees that the QI will have the largest possible contrast (i.e., $0 \leqslant \rho_{33} \leqslant 8 \alpha^{2} Q_{o}^{2}$ ), and also that the maximum of the signal (proportional to $\rho_{33}$ ) will be as large as possible. Recalling that Eq. (58) has been derived under the assumption that $X_{o} \gg X_{q}>X_{q 1}$, we see that the above optimization is subject to the constraint $2 \alpha Q_{o}$ $\gg|M| v(\theta)$, which, combined with our requirement that $|M| v(\theta) \approx Q_{o}$, leads to the conclusion that for optimum experimental conditions we should have $2 \alpha \gg 1$, or equivalently $\sqrt{\gamma_{2} / \gamma_{3}} \gg 0.5$. Hence the choice of the atom to use in this particular type of experiment is crucial, since the accuracy of the observation is ultimately determined by the atomic decay rates, and in particular by the ratio of $\gamma_{2}$ to $\gamma_{3}$. In our experimental work of Ref. [10], $\sqrt{\gamma_{2} / \gamma_{3}} \simeq 1.29$.

Turning now to the size of the modulation in Eq. (58), we note that it can be characterized by the visibility $V$ as defined by

$$
V \equiv \frac{\left(\rho_{33}\right)_{\max }-\left(\rho_{33}\right)_{\min }}{\left(\rho_{33}\right)_{\max }+\left(\rho_{33}\right)_{\min }} .
$$

Assuming $N_{1}=N_{2} \equiv N$, we have that, for quantum squeezing, $N<|M| \leqslant \sqrt{N(N+1)}$, where for classical states $|M|$ $<N$. Hence, $N / Q_{o}<V_{\text {squeezed }} \leqslant \sqrt{N(N+1)} / Q_{o}$ and $V_{\text {classical }}$ $\leqslant N / Q_{o}$ for the cases of squeezed and classical field excitation, respectively, with fundamentally different functional dependences. In particular, for the case of quantum squeezing in minimum uncertainty states, $V_{\text {squeezed }}(N \rightarrow 0)$ $\rightarrow \sqrt{N} / Q_{o}$ which has a characteristic square-root dependence on $N$. Observation of this dependence would constitute an unambiguous quantum effect of the same nature as that of Ref. [8]. As a matter of fact, this type of experiment has certain advantages over that of Ref. [8], where we sought an
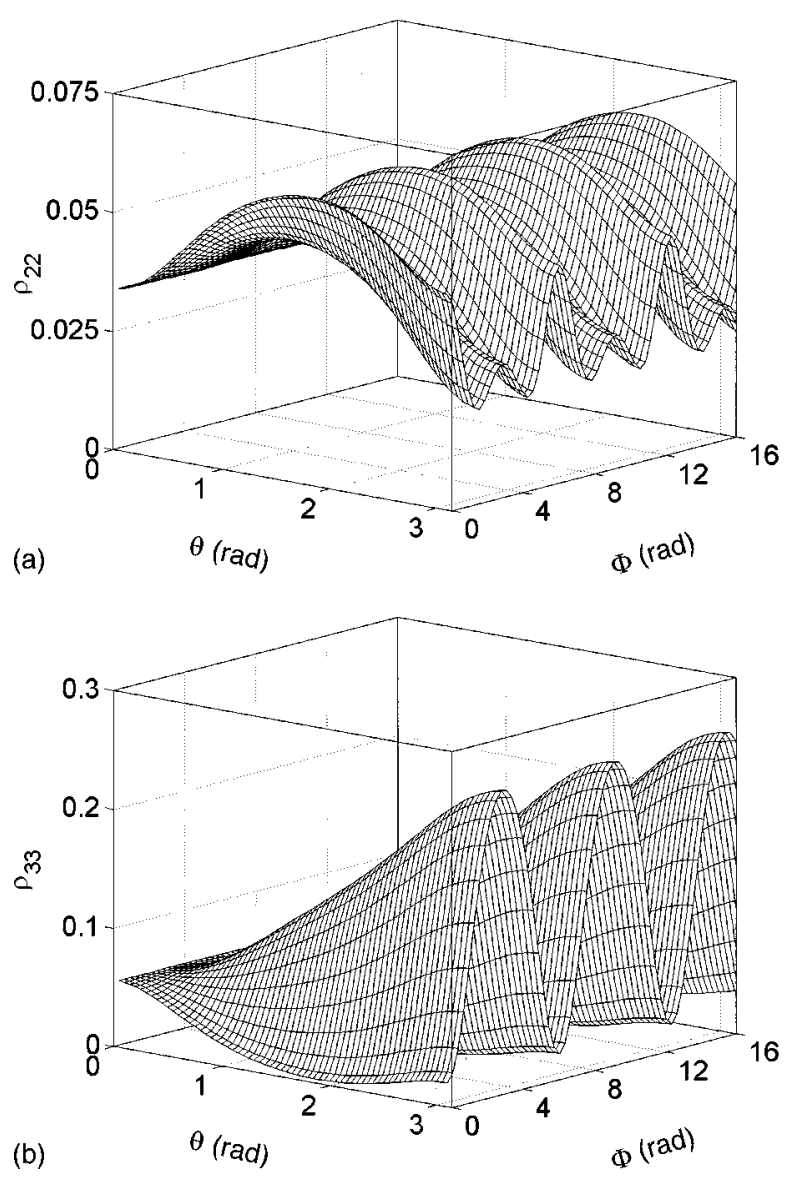

FIG. 2. Atomic populations (a) $\rho_{22}$ and (b) $\rho_{33}$ vs the focusing angle of squeezing $\theta$ and the quantum interference angle $\Phi$ plotted for $\quad \alpha \equiv \sqrt{\gamma_{2} / \gamma_{3}}=\sqrt{5 / 3}, \quad Q_{o}=0.1, \quad N_{1}=N_{2}=0.1, \quad$ and $|M|=\sqrt{N(N+1)}$.

asymptotically linear dependence for the two-photon excitation versus the intensity of the incident squeezed vacuum for the case of small $N$. In particular, the signal for the experiment proposed here, the distinctive functional form of $\sqrt{N}$, could be easier to identify as compared to the linear functional form $N$, which can have other origins if necessary precautions are not taken (e.g., the scattered background from the squeezed field also scales as $N$ ). Last but not least, the actual data acquisition (photocounting) could be viewed in the frequency domain, where, as in Ref. [10], one looks at the Fourier transform of the photocurrent, in this way reducing the background content at the observation frequency and improving the signal-to-noise ratio. Note that for a photoncounting experiment, with $Q_{o}=0$ as in Ref. [8], one detects a signal given by the term $X_{q}^{2}=\left(|M|^{2} \alpha^{2}+N_{1} N_{2}\right) v^{2}(\theta)$ in Eq. (56). By contrast, with $Q_{o}$ nonzero, there is "mixing" gain so that $X_{q} X_{0}, X_{0}^{2} \gg X_{q}^{2}$. The operational advantage is that the quantum noise (photon counting associated with $X_{0}^{2}$ ) can then be dominant over other noises such as dark counts in the photodetectors and scattered light, which were limiting factors in the experiment of Ref. [8].

\section{SQUEEZING OVER A LARGE SOLID ANGLE}

By increasing the angle of focusing of the squeezed light onto the atoms, the value of $v(\theta)$ increases, and additional 

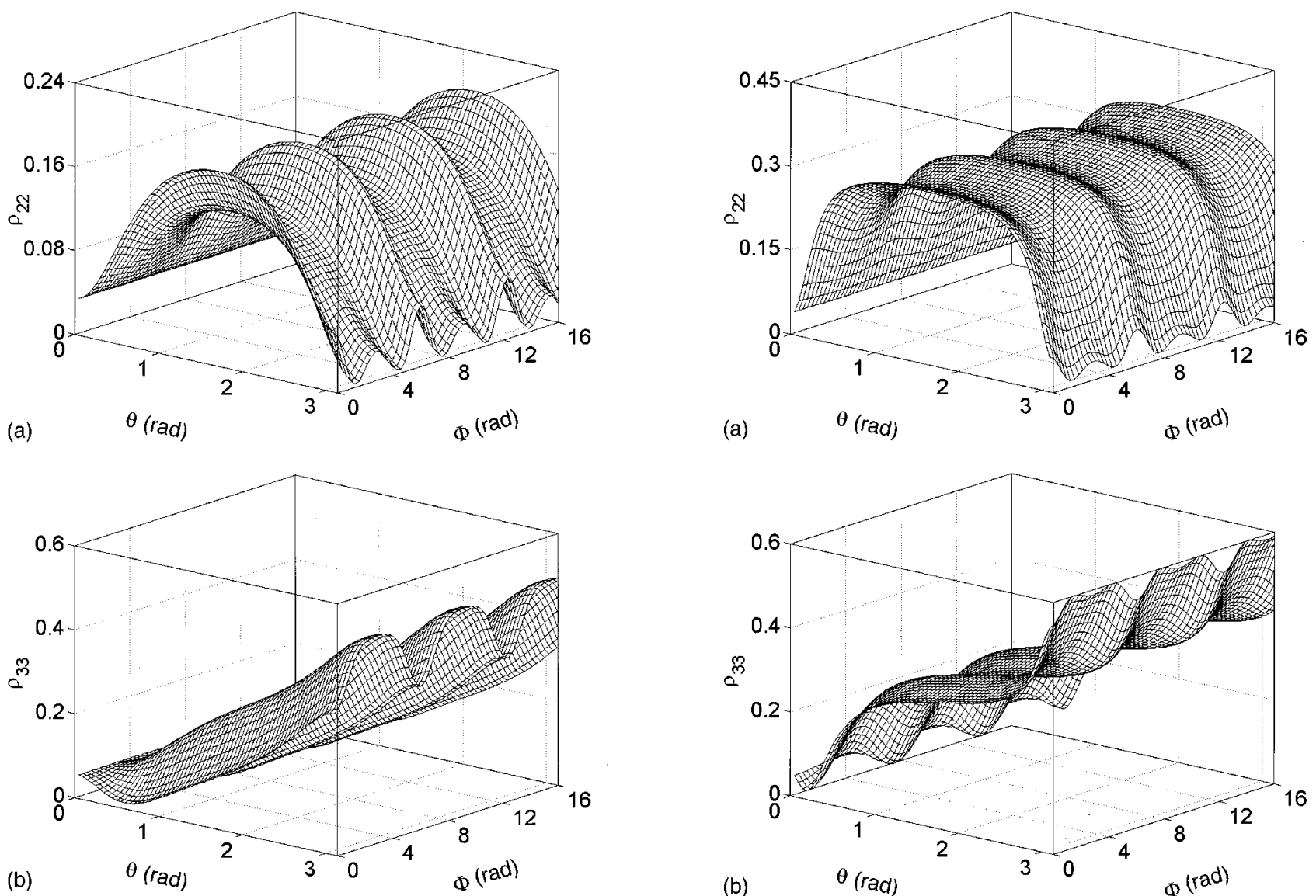

FIG. 3. Atomic populations (a) $\rho_{22}$ and (b) $\rho_{33}$ vs the focusing angle of squeezing $\theta$ and the quantum interference angle $\Phi$ plotted for $\alpha \equiv \sqrt{\gamma_{2} / \gamma_{3}}=\sqrt{5 / 3}, \quad Q_{o}=0.1, \quad N_{1}=N_{2}=1, \quad$ and $|M|=\sqrt{N(N+1)}$.

terms in the expansion of $v(\theta)$ in Eqs. (55) and (56) must be retained for the solution to be accurate. Figures 2-4 show graphs of the full solution for the excited-state populations $\rho_{22}$ and $\rho_{33}$ as a function of the focusing angle $\theta$ and of the quantum interference phase $\Phi$. Clearly, along the $\Phi$ axis we observe the $2 \pi$-periodic structure ( $\pi$ periodic in the phase $\phi_{o}$ of the RO) of the atomic populations, a direct consequence of quantum interference, while along the $\theta$ dimension we see increasingly interesting features that appear as the solid angle of coverage is increased. In particular, the effects of QI become much more profound for large focusing angles; for example, for certain $\Phi$ in Figs. 3 and 4 it leads to complete suppression of the two-excited-state population $\rho_{22}$. To elaborate more on this point, assuming complete coverage of the atoms by the squeezing (i.e., $\theta=\pi$ ) it can be shown from Eqs. (52) and (53) that for the particular choice of $\Phi=\pi / 2$, $N_{s}=N_{i}=N=\left[1 /\left(\alpha^{2}-1\right)\right]$, and $|M|=\sqrt{N(N+1)}$,

$$
\rho_{22}=0
$$

and

$$
\rho_{33}=\frac{1}{2} \text {. }
$$

It is noted that this interesting case only occurs for atomic systems with linewidths satisfying $\gamma_{2}>\gamma_{3}$, since $N$ has to be a positive number.

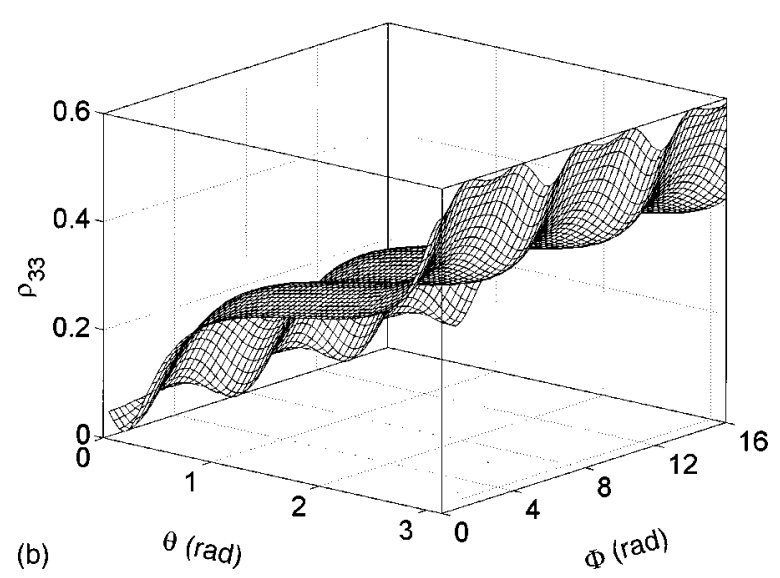

FIG. 4. Atomic populations (a) $\rho_{22}$ and (b) $\rho_{33}$ vs the focusing angle of squeezing $\theta$ and the quantum interference angle $\Phi$ plotted for $\quad \alpha \equiv \sqrt{\gamma_{2} / \gamma_{3}}=\sqrt{5 / 3}, \quad Q_{o}=0.1, \quad N_{1}=N_{2}=10, \quad$ and $\quad|M|$ $=\sqrt{N(N+1)}$.

The particular choice of parameters for Figs. 2-4 is such as to model the atomic system studied in Ref. [10], for which $\alpha \approx \sqrt{5 / 3}$, and where the intensity of the RO was $Q_{o} \approx 0.1$. Recall that the theory of Eqs. (52) and (53) was developed under the assumption of weak RO fields, for which Stark shifts caused by these can be neglected and hence we limit $Q_{o} \ll 1$. Nevertheless, inclusion of the Stark shifts due to the $Q_{o}$ field only requires the use of the full expression for $A$ [defined in Eq. (44)], which simply changes the definition of the quantities $\Xi_{1}, \Xi_{2}, \Lambda_{1}$, and $\Lambda_{2}$ in Eqs. (47)-(50).

Note that in the experiment [10] the focusing angle was very small $\theta \approx 0.1 \mathrm{rad}$, and hence only simple sinusoidal oscillations were present rather than the more complex interference patterns that appear for higher values of $\theta$ as shown on Figs. 2-4. In the examples presented here, we have assumed a minimum uncertainty state with $|M|^{2}=N(N+1)$, where $N \equiv N_{1}=N_{2}$, and successive figures have been drawn for increasingly stronger squeezed fields. There, for Fig. 2 we assume $N=0.1$, for Fig. 3 we use $N=1$, and for Fig. 4 we use $N=10$. Note that $M$ and $N$ refer to the squeezed field at the source, with the factor $v(\theta)$ accounting for "alterations" in coupling the source to the atoms. We also note that by changing the value of $\alpha$, the corresponding graphs will take both quantitatively as well as qualitatively different forms than shown in Figs. 2-4. 


\section{A. Phase sensitivity of atomic populations and quantum optical effects}

As previously noted, the atomic populations $\rho_{22}$ and $\rho_{33}$, exhibit phase sensitivity via the dependencies expressed in Eqs. (52) and (53) on the term $Q_{o}|M| \cos \Phi$ [here we have set $v(\theta)=1]$. Hence both $Q_{o}$ and $M$ must be nonzero in order to have phase-sensitive atomic populations. In the case of thermal excitation for which $M=0$ and the signal and idler fields are each in a thermal state with occupation number given by $N_{1}$ and $N_{2}$, respectively, there is no phase sensitivity, and the atomic populations can be shown to be equal to

$$
\rho_{22}^{\mathrm{th}}=\frac{\left(N_{1} \alpha^{2}+N_{2}+1\right)\left(N_{2} N_{1}+N_{1}+4 Q_{o}^{2}\right)}{4 Q_{o}^{2}\left(1+3 N_{1} \alpha^{2}+3 N_{2}+2 \alpha^{2}\right)+\left(1+N_{2}+3 N_{2} N_{1}+2 N_{1}\right)\left(N_{1} \alpha^{2}+N_{2}+1\right)}
$$

and

$$
\rho_{33}^{\text {th }}=\frac{4 Q_{o}^{2}\left(N_{1} \alpha^{2}+N_{2}+\alpha^{2}\right)+\left(N_{1} \alpha^{2}+N_{2}+1\right) N_{2} N_{1}}{4 Q_{o}^{2}\left(1+3 N_{1} \alpha^{2}+3 N_{2}+2 \alpha^{2}\right)+\left(1+N_{2}+3 N_{2} N_{1}+2 N_{1}\right)\left(N_{1} \alpha^{2}+N_{2}+1\right)} .
$$

Note that by setting $Q_{o}=0$ we recover Eqs. (12) and (13) of Ref. [5(b)].

Next we turn to the case $M \neq 0$, which implies phase sensitivity but does not necessarily imply a nonclassical effect. In particular, one should compare the minimum uncertainty quantum states for which $|M|^{2}=N(N+1)$ with the "closest" classical states, namely, the classical squeezed states for which $|M|^{2}=N^{2}$ (here, as above, we make the simplifying assumption that $\left.N_{1}=N_{2} \equiv N\right)$. In both cases, we have phase-sensitive atomic populations. In order to identify intrinsically quantum effects associated with the nonclassical nature of the quantum squeezed vacuum, we concentrate on the region of small $N \leq 1$, where the distinction between the effects of classical and quantum squeezing is maximum. In particular, if we assume that $N \ll 1$, we can expand the solutions for the atomic populations $\rho_{22}$ and $\rho_{33}$ as given by Eqs. (52) and (53) in powers of $N$, which leads to

$$
\rho_{22}^{q}=\frac{1}{\left(1+2 \alpha^{2}\right)+\frac{1}{4 Q_{o}^{2}}}\left[1+\frac{\alpha^{2}+1}{\left(1+2 \alpha^{2}\right)+\frac{1}{4 Q_{o}^{2}}} \frac{\sqrt{N}}{Q_{o}} \cos \Phi\right]+O(N)
$$

and

$$
\rho_{33}^{q}=\frac{\alpha^{2}}{\left(1+2 \alpha^{2}\right)+\frac{1}{4 Q_{o}^{2}}}\left[1-\frac{\alpha^{2}+\frac{1}{4 Q_{o}^{2}}}{\left(1+2 \alpha^{2}\right)+\frac{1}{4 Q_{o}^{2}}} \frac{\sqrt{N}}{Q_{o}} \cos \Phi\right]+O(N)
$$

for the case of excitation with quantum squeezing. For the excitation with classical squeezing, we obtain

$$
\rho_{22}^{\mathrm{cl}}=\frac{1}{\left(1+2 \alpha^{2}\right)+\frac{1}{4 Q_{o}^{2}}}\left[1+\frac{\left(1+\alpha^{2}\right)}{\left(1+2 \alpha^{2}\right)+\frac{1}{4 Q_{o}^{2}}} \frac{N}{Q_{o}} \cos \Phi\right]+\frac{32 Q_{o}^{4}\left(\alpha^{4}-1\right)+8 Q_{o}^{2}\left(\alpha^{2}-1\right)+1}{16 Q_{o}^{4}\left(\left(1+2 \alpha^{2}\right)+\frac{1}{4 Q_{o}^{2}}\right)} N+O\left(N^{2}\right)
$$

and

$$
\rho_{33}^{\mathrm{cl}}=\frac{\alpha^{2}}{\left(1+2 \alpha^{2}\right)+\frac{1}{4 Q_{o}^{2}}}\left[1-\frac{\alpha^{2}+\frac{1}{4 Q_{o}^{2}}}{\left(1+2 \alpha^{2}\right)+\frac{1}{4 Q_{o}^{2}}} \frac{N}{Q_{o}} \cos \Phi\right]-\frac{4 Q_{o}^{2}\left(\alpha^{4}-1\right)+\left(\alpha^{4}+3 \alpha^{2}-1\right)}{4 Q_{o}^{2}\left(\left(1+2 \alpha^{2}\right)+\frac{1}{4 Q_{o}^{2}}\right)^{2}} N+O\left(N^{2}\right) .
$$

Equations (64)-(67) show that for small $N$, phasesensitive modulation onsets for the case of quantum squeezing proportionally to $\sqrt{N} / Q_{o}$, while for the case of classical squeezing the onset of the modulation is proportional to $N / Q_{o}$, which is of higher order in $N$. This again raises the issue of the functional dependance of the modulation size versus $N$. The distinction between the functional forms $\sqrt{N}$ and $N$ is apparently an unambiguous signature of quantum effects.

For completeness, we note that in the limit $N_{1}=N_{2}=N$ 

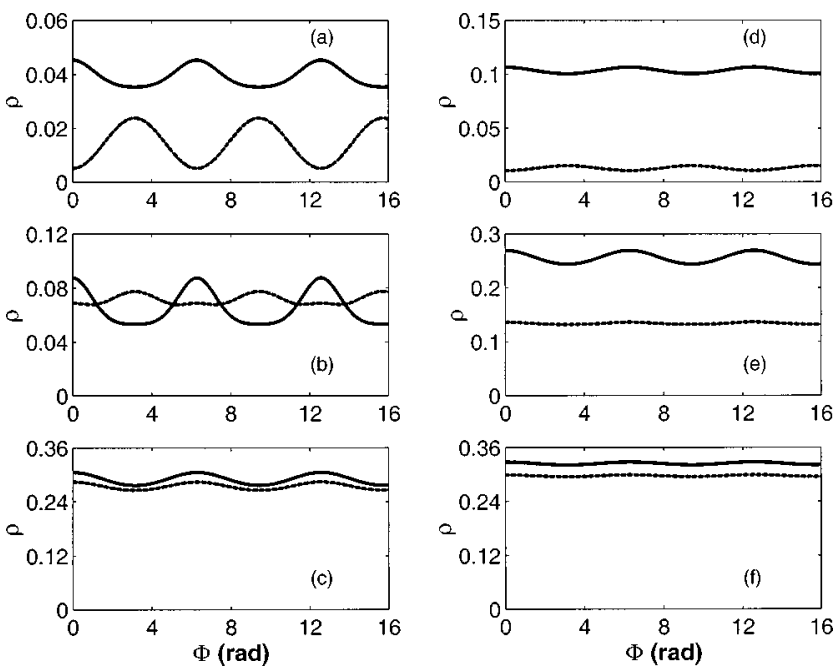

FIG. 5. Atomic populations: (-) solid lines are for $\rho_{22}$; (. . . .) dotted lines are for $\rho_{33}$. Here $\alpha \equiv \sqrt{\gamma_{2} / \gamma_{3}}=\sqrt{1 / 10}$ and $Q_{o}=0.1 . N=0.1$ for $\left(\mathrm{a}_{\mathrm{sq}}\right)$ and $\left(\mathrm{a}_{\mathrm{cl}}\right), N=1$ for $\left(\mathrm{b}_{\mathrm{sq}}\right)$ and $\left(\mathrm{b}_{\mathrm{cl}}\right)$, and $N=10$ for $\left(\mathrm{c}_{\mathrm{sq}}\right)$ and $\left(\mathrm{c}_{\mathrm{cl}}\right)$. Two cases of excitation with quantum squeezed light $[|M|=\sqrt{N(N+1)}]\left[\left(\mathrm{a}_{\mathrm{sq}}\right),\left(\mathrm{b}_{\mathrm{sq}}\right)\right.$, and $\left.\left(\mathrm{c}_{\mathrm{sq}}\right)\right]$ and classical squeezed light $(|M|=N)\left[\left(\mathrm{a}_{\mathrm{cl}}\right),\left(\mathrm{b}_{\mathrm{cl}}\right)\right.$, and $\left.\left(\mathrm{c}_{\mathrm{cl}}\right)\right]$ have been considered.

$\ll 1$, expansions of Eqs. (62) and (63), for excitation with thermal fields, results in

$$
\begin{aligned}
\rho_{22}^{\mathrm{th}}= & \frac{1}{\left(1+2 \alpha^{2}\right)+\frac{1}{4 Q_{o}^{2}}}+\frac{32 Q_{o}^{4}\left(\alpha^{4}-1\right)+8 Q_{o}^{2}\left(\alpha^{2}-1\right)+1}{16 Q_{o}^{4}\left(\left(1+2 \alpha^{2}\right)+\frac{1}{4 Q_{o}^{2}}\right)^{2}} N \\
& +O\left(N^{2}\right)
\end{aligned}
$$

and
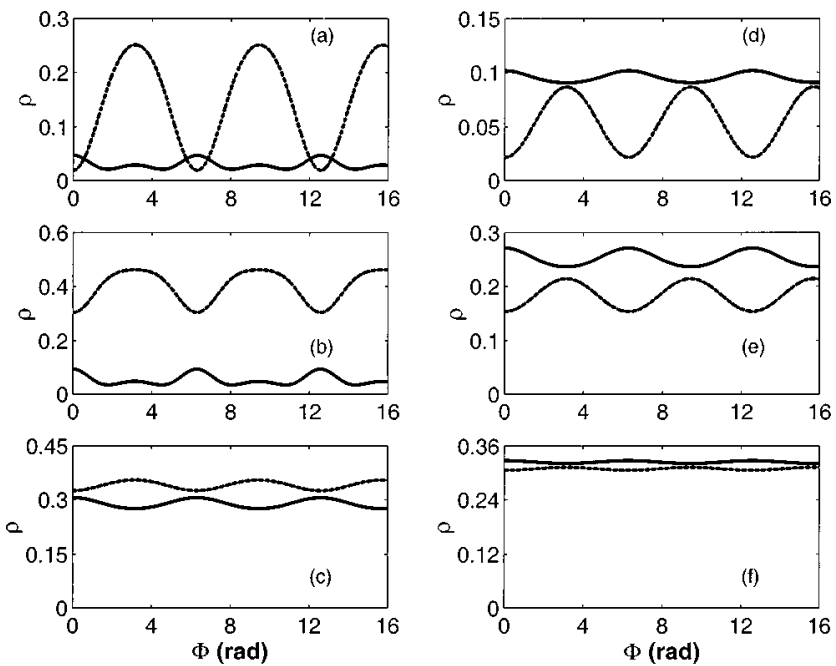

FIG. 6. Atomic populations: (-) solid lines are for $\rho_{22}$; (. . . .) dotted lines are for $\rho_{33}$. Here $\alpha \equiv \sqrt{\gamma_{2} / \gamma_{3}}=\sqrt{5 / 3}$ and $Q_{o}=0.1 . N=0.1$ for $\left(\mathrm{a}_{\mathrm{sq}}\right)$ and $\left(\mathrm{a}_{\mathrm{cl}}\right), N=1$ for $\left(\mathrm{b}_{\mathrm{sq}}\right)$ and $\left(\mathrm{b}_{\mathrm{cl}}\right)$; and $N=10$ for $\left(\mathrm{c}_{\mathrm{sq}}\right)$ and $\left(\mathrm{c}_{\mathrm{cl}}\right)$. Two cases of excitation with quantum squeezed light $[|M|=\sqrt{N(N+1)}]\left[\left(\mathrm{a}_{\mathrm{sq}}\right),\left(\mathrm{b}_{\mathrm{sq}}\right)\right.$, and $\left.\left(\mathrm{c}_{\mathrm{sq}}\right)\right]$ and classical squeezed light $(|M|=N)\left[\left(\mathrm{a}_{\mathrm{cl}}\right),\left(\mathrm{b}_{\mathrm{cl}}\right)\right.$, and $\left.\left(\mathrm{c}_{\mathrm{cl}}\right)\right]$ have been considered.
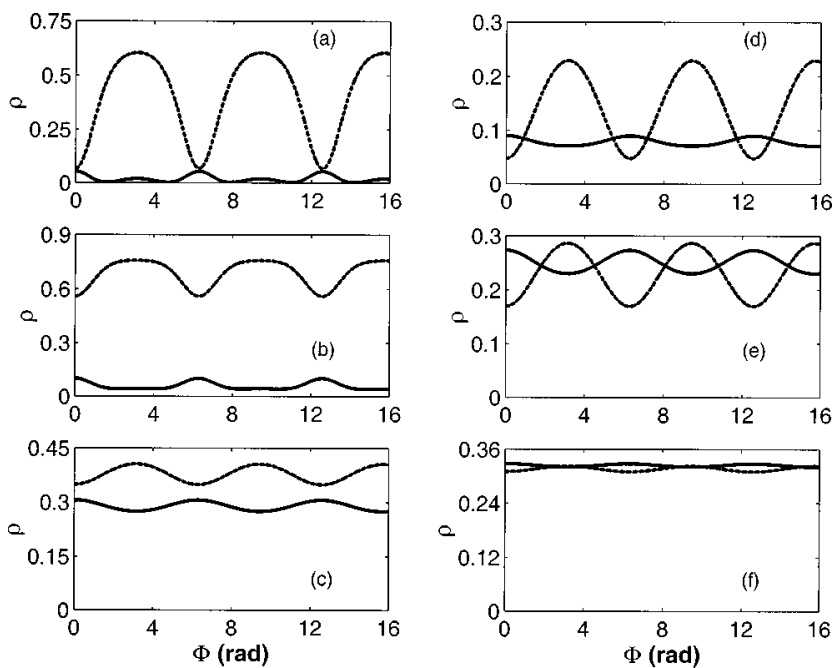

FIG. 7. Atomic populations: (-) solid lines are for $\rho_{22}$; (. . . .) dotted lines are for $\rho_{33}$. Here $\alpha \equiv \sqrt{\gamma_{2} / \gamma_{3}}=\sqrt{10 / 1}$ and $Q_{o}=0.1 . N=0.1$ for $\left(\mathrm{a}_{\mathrm{sq}}\right)$ and $\left(\mathrm{a}_{\mathrm{cl}}\right), N=1$ for $\left(\mathrm{b}_{\mathrm{sq}}\right)$ and $\left(\mathrm{b}_{\mathrm{cl}}\right)$, and $N=10$ for $\left(\mathrm{c}_{\mathrm{sq}}\right)$ and $\left(\mathrm{c}_{\mathrm{cl}}\right)$. Two cases of excitation with quantum squeezed light $[|M|=\sqrt{N(N+1)}]\left[\left(\mathrm{a}_{\mathrm{sq}}\right),\left(\mathrm{b}_{\mathrm{sq}}\right)\right.$, and $\left.\left(\mathrm{c}_{\mathrm{sq}}\right)\right]$ and classical squeezed light $(|M|=N)\left[\left(\mathrm{a}_{\mathrm{cl}}\right),\left(\mathrm{b}_{\mathrm{cl}}\right)\right.$, and $\left.\left(\mathrm{c}_{\mathrm{cl}}\right)\right]$ have been considered.

$$
\begin{aligned}
\rho_{33}^{\text {th }}= & \frac{\alpha^{2}}{\left(1+2 \alpha^{2}\right)+\frac{1}{4 Q_{o}^{2}}}-\frac{4 Q_{o}^{2}\left(\alpha^{4}-1\right)+\left(\alpha^{4}+3 \alpha^{2}-1\right)}{4 Q_{o}^{2}\left(\left(1+2 \alpha^{2}\right)+\frac{1}{4 Q_{o}^{2}}\right)^{2}} N \\
& +O\left(N^{2}\right),
\end{aligned}
$$

which contain the same terms as $\rho_{22}^{\mathrm{cl}}$ and $\rho_{33}^{\mathrm{cl}}$ as in Eqs. (66) and (67), but without the modulation.

Figures 5-7 show examples of the quantum interference patterns that are predicted from our theory [Eqs. (52) and (53)] (without the assumption that $N \ll 1$ ) for an extensive set of parameters. For each figure we choose a different value for $\alpha$, with $\alpha=\sqrt{1 / 10}$ for Fig. 5, $\alpha=\sqrt{5 / 3}$ for Fig. 6, and $\alpha=\sqrt{10 / 1}$ for Fig. 7. According to the definition of $\alpha[\mathrm{Eq}$. (39)], we note that these values correspond to the ratios of the atomic linewidths equal to $\gamma_{2} / \gamma_{3}=0.1,1.67$, and 10 for Figs. 5, 6, and 7, respectively. With other parameters kept the same, it is clear that in the first case with $\gamma_{2} / \gamma_{3}=0.1$ the atomic population of the second excited level would be in general greater than the atomic population of the third excited level, $\rho_{22}>\rho_{33}$, while in the third example with $\gamma_{2} / \gamma_{3}=10$ the opposite must be true, as is evident from Figs. 7. In addition, each figure has been plotted for three different values of $N$, while $Q_{o}=0.1$ has been kept constant throughout. For each of these cases we show the atomic populations $\rho_{22}$ and $\rho_{33}$ for excitation with minimum uncertainty quantum squeezing $|M|^{2}=N(N+1)$ and for classical squeezing $|M|^{2}=N^{2}$.

\section{B. Quantum optical effects in phase-sensitive inversion}

Another interesting feature of the atomic system under consideration is the fact that it exhibits phase-sensitive inver- 
sion. This subject is of particular interest because, as in Ref. [28], the phase sensitivity is induced by squeezed light. For the purposes of the present discussion we find it convenient to define a measure of the inversion between the $|i\rangle$ and $|j\rangle$ states in terms of the ratio of the populations $\rho_{i i}$ and $\rho_{j j}$ in these two states according to

$$
I_{i j} \equiv \frac{\rho_{i i}}{\rho_{j j}} .
$$

Once more making the simplified assumption that $N_{1}=N_{2} \equiv N$ leads to the following expression for the population inversion between the three- and two-excited levels:

$$
I_{32} \equiv \frac{\rho_{33}}{\rho_{22}}=\frac{\left(4 \frac{N\left(\alpha^{2}+1\right)+\alpha^{2}}{N\left(\alpha^{2}+1\right)+1}-\Psi^{2} \sin ^{2} \Phi\right) Q_{o}^{2}-\alpha \Psi Q_{o} \cos \Phi-|M|^{2} \frac{N\left(\alpha^{2}+1\right)-\alpha^{2}}{N\left(\alpha^{2}+1\right)+1}+N^{2}}{\left(4-\Psi^{2} \sin ^{2} \Phi\right) Q_{o}^{2}+N(N+1)-|M|^{2}},
$$

where

$$
\Psi \equiv \frac{4 \alpha|M|}{N\left(\alpha^{2}+1\right)+1} .
$$

This expression takes fundamentally different forms depending on the statistics of the signal and idler fields used for the excitation. In particular, for minimum uncertainty squeezed states, $|M|^{2}=N(N+1)$, we find

$$
I_{32}^{q}=\frac{\left(4 \frac{N\left(\alpha^{2}+1\right)+\alpha^{2}}{N\left(\alpha^{2}+1\right)+1}-\Psi_{q}^{2} \sin ^{2} \Phi\right) Q_{o}^{2}-\alpha \Psi_{q} Q_{o} \cos \Phi+\frac{N \alpha^{2}}{N\left(\alpha^{2}+1\right)+1}}{\left(4-\Psi_{q}^{2} \sin ^{2} \Phi\right) Q_{o}^{2}},
$$

while for classically squeezed states $|M|^{2}=N^{2}$ we have

$$
I_{32}^{\mathrm{cl}}=\frac{\left(4 \frac{N\left(\alpha^{2}+1\right)+\alpha^{2}}{N\left(\alpha^{2}+1\right)+1}-\Psi_{\mathrm{cl}}^{2} \sin ^{2} \Phi\right) Q_{o}^{2}-\alpha \Psi_{\mathrm{cl}} Q_{o} \cos \Phi+\frac{N^{2}\left(\alpha^{2}+1\right)}{N\left(\alpha^{2}+1\right)+1}}{\left(4-\Psi_{\mathrm{cl}}^{2} \sin ^{2} \Phi\right) Q_{o}^{2}+N},
$$

and for thermal states $|M|^{2}=0$ we obtain

$$
I_{32}^{\mathrm{th}}=\frac{4 \frac{N\left(\alpha^{2}+1\right)+\alpha^{2}}{N\left(\alpha^{2}+1\right)+1} Q_{o}^{2}+N^{2}}{4 Q_{o}^{2}+N(N+1)} .
$$

Here, from Eq. (72), $\Psi_{q}$ and $\Psi_{\mathrm{cl}}$ are defined to be

$$
\Psi_{q} \equiv \frac{4 \alpha \sqrt{N(N+1)}}{N\left(\alpha^{2}+1\right)+1}
$$

and

$$
\Psi_{\mathrm{cl}} \equiv \frac{4 \alpha N}{N\left(\alpha^{2}+1\right)+1} .
$$

Note that in the cases described above, the mean photon flux as given by $N$ is the same, with only the form of $M$ changing from one expression to the other. Also observe that the inversion is phase sensitive for both quantum and classical squeezed states, but not for thermal fields. As before, in order to differentiate between classical $|M|=N$ and quantum $|M|=\sqrt{N(N+1)}$ effects, we must concentrate on the region where the difference is the largest, which is for small $N$ $\lesssim 1$. Assuming $N \ll 1$ and $N<Q_{o}$, allows us to expand Eqs. (73) and (74) in powers of $N$, leading to

$$
I_{32}^{q}=\alpha^{2}-\alpha^{2} \frac{\sqrt{N}}{Q_{o}} \cos \Phi+O(N)
$$

for the case of excitation with quantum squeezing, while for excitation with classical squeezing we obtain that

$$
I_{32}^{\mathrm{cl}}=\alpha^{2}+\left(1-\alpha^{4}-\frac{\alpha^{2}}{4 Q_{o}^{2}}\right) N-\alpha^{2} \frac{N}{Q_{o}} \cos \Phi+O\left(N^{2}\right) .
$$

Again, for the purposes of completeness, expansion of Eq. (75) gives

$$
I_{32}^{\text {th }}=\alpha^{2}+\left(1-\alpha^{4}-\frac{\alpha^{2}}{4 Q_{o}^{2}}\right) N+O\left(N^{2}\right)
$$

for the case of thermal fields.

The ratio of the atomic linewidths $\alpha$ plays a key role in the determination of the values the inversion takes. In par- 
ticular, if we turn off the quantum (or classical) field (i.e., set $N=M=0)$ then $I_{32}^{q}=I_{32}^{\mathrm{cl}}=I_{32}^{\mathrm{th}}=\alpha^{2}$, which, when combined with the definition of the inversion $I_{32}=\rho_{33} / \rho_{22}$ and the definition of $\alpha=\sqrt{\gamma_{2} / \gamma_{3}}$, leads to $\rho_{33} \gamma_{3}=\rho_{22} \gamma_{2}$. This is what we would naturally expect from the atomic rates of spontaneous emission when the system is driven only by the RO field $Q_{o}$.

Returning now to the discussion of how to distinguish between intrinsically quantum versus classical effects, we observe that Eq. (78) predicts that even for arbitrarily small driving fields (i.e., $N \ll 1$ and $Q_{o} \ll 1$ ), and as long as $\sqrt{N} / Q_{o} \approx 1$, we can obtain phase-sensitive inversion that varies between the extreme values $0 \leqslant I_{32}^{q} \leqslant 2 \alpha^{2}$. In other words, the correlations of the fluctuations of the nonclassical field $\hat{\varepsilon}_{q}$ as expressed by $M$, and the QI of the excitation amplitudes, can lead to a complete suppression of the excited-state population $\left(I_{32}^{q}=0\right)$, or, in the opposite extreme, to population inversion twice as large as the usual inversion $\left(I_{32}^{q}\right.$ $=2 \alpha^{2}$ ). Hence the effect of phase-sensitive inversion can be viewed as a modification of the relative strength of the spontaneous emission rates for the $3 \rightarrow 2$ and $2 \rightarrow 1$ transitions, where, for complete suppression of the excited state population, $\alpha \rightarrow \alpha_{\text {eff }}=0$; however in the opposite extreme $\alpha \rightarrow \alpha_{\text {eff }}$ $=\sqrt{2} \alpha$ (recall that $\alpha=\sqrt{\gamma_{2} / \gamma_{3}}$ ). Similar effects of QI and modification of the effective decay rates have been extensively studied in the context of lasing without inversion [26]. However, in our case we have a "new player" on the scene, namely, the quantum statistics of the exciting fields as described by the correlations of the quadrature fluctuations given by $M$.

The above qualitative observations, although not unique to quantum squeezing [since Eq. (79) implies that the inversion modulates above and below the value $\alpha^{2}$ even with classical squeezing (i.e., $M=N$ )], do, however, contain quantitatively distinctive differences between the cases of excitation with classical versus quantum squeezing. In particular, Eq. (79) implies that in the same limiting case as we have considered above, (i.e., $N \ll 1$ and $Q_{o} \ll 1$ ), and assuming that the value of $\alpha$ is not very large (i.e., $\alpha \ll 1 / Q_{o}$ ), the population inversion between the three- and two-excited states is roughly constant, $I_{32}^{\mathrm{cl}} \simeq \alpha^{2}\left[1-\left(N / 4 Q_{o}^{2}\right)\right] \approx \frac{3}{4} \alpha^{2}$ (assuming as above that $\left.\sqrt{N} / Q_{o} \approx 1\right)$. Hence we have a very strong prediction that will distinguish between intrinsically quantum versus classical effects in a much more profound way than the difference in functional form that was mentioned above, or that which was studied in Ref. [8], namely, that excitation of the atoms by a combination of a very weak quantum field $\hat{\varepsilon}_{q}(M=\sqrt{N(N+1)}, N \ll 1)$ and a very weak classical RO field $\hat{\varepsilon}_{0}\left(Q_{o} \ll 1\right)$ will lead to phase-sensitive inversion between the three- and two-excited levels that could range between 0 and $2 \alpha^{2}$. This is in sharp contrast to excitation by classical squeezed fields, for which the phase sensitivity of the inversion disappears, and which, within the limits of this calculation, will have a value roughly equal to $\frac{3}{4} \alpha^{2}$. The price to pay is that we have limited ourselves to very weak excitation fields, and hence the excited-state populations which ultimately determine the size of the signal to be observed are also very weak, which reduces the signalto-noise ratio and makes the experiment more difficult. Nevertheless, this is a good technique for observing intrinsically quantum effects associated with the interaction of nonclassical states of the electromagnetic field with atoms, which very distinctively distinguishes between classical and quantum effects, and which persists even for very small focusing angles of the squeezed vacuum onto the atoms $[N \rightarrow N v(\theta) \ll 1]$.

For completeness, we note that for the case of thermal excitation the population inversion in the above limit of weak fields reduces to $I_{32}^{\text {th }} \simeq \alpha^{2}\left[1-\left(N / 4 Q^{2}\right)\right]$, which is the same limit as for classical squeezing. In other words, the case of very weak classical squeezed fields resembles the case of thermal fields, and loses its squeezing character (scales as $N$ ), while as we have seen that in the case of quantum squeezing the effects of squeezing persist (scale as $\sqrt{N}$ ).

\section{CONCLUSION}

In this paper we have presented a solution based on the quantum master equation for the problem of two-photon excitation of a three-level atom by a combination of the signal and idler output fields from a ND-OPO and a strong coherent RO field. As a result of the combined excitation, and due to the presence of multiple excitation pathways, the atomic populations exhibit phase sensitivity to the relative phase of the fields, and in particular to the asymmetric distribution of fluctuations for a squeezed state. We have derived analytic expressions for the excited-state populations $\rho_{22}$ and $\rho_{33}$, and have analyzed several examples of quantum interference in the system including the effects of finite angle of focusing of the squeezed field onto the atoms, which is a crucial experimental constraint. Particular emphasis was given to identifying intrinsically quantum effects associated with the nonclassical nature of the squeezed vacuum, with specific comparison to the closest classical analog of quantum squeezing, for which similar qualitative but different quantitative effects can be observed. Additional comparisons to the simpler case where the signal and idler fields are in a thermal state have been presented. Finally, we have analyzed population inversion in our system, which was shown to be phase sensitive due to QI, and demonstrated that for weak excitation there are distinct differences between excitation with quantum versus classical squeezing.

One notable feature of the class of nonclassical phenomena that we have considered here is that they manifest for small intensities of the squeezed field, and in fact they become more pronounced as this intensity decreases. This is in contrast with a different class of phenomena (such as subnatural line narrowing [1]) for which the nonclassical nature of the effects associated with the interaction of nonclassical states of the electromagnetic field with atoms becomes more pronounced for strong intensities of the squeezed light. However, contrary to the latter case, the phenomena we have discussed in this paper are persistent even for very small coupling efficiencies of the squeezed light with the atoms. This is a crucial feature from an experimentalist point of view, and the observation of any of the nonclassical effects discussed in the literature almost always rely on the ability to realize these effects with very small coupling efficiencies. Therefore, effects which persist independently of coupling 
efficiency are extremely interesting, and so far have been the only type of effects that have been observed [8]. Notice that as the coupling efficiency increases, which in our case is equivalent to $v(\theta) \rightarrow 1$, effects of the second class will also start to manifest themselves, and one needs to take into account more carefully the full expression of $\rho_{33}$, as well as to consider the frequency spectra, in order to unveil additional signatures of the nonclassical interaction of the squeezed field with the atoms.

\section{ACKNOWLEDGMENTS}

This research was supported by the Division of Chemical Science, Office of Basic Energy Science, Office of Energy Research, U.S. Department of Energy.
[1] C. W. Gardiner, Phys. Rev. Lett. 56, 1917 (1986).

[2] For a review see A. S. Parkins, in Modern Nonlinear Optics, edited by M. Evans and S. Kielich (Wiley, New York, 1993).

[3] J. Gea-Banacloche, Phys. Rev. Lett. 62, 1603 (1989).

[4] J. Javaninen and P. L. Gould, Phys. Rev. A 41, 5088 (1990).

[5] (a) Z. Ficek and P. D. Drummond, Phys. Rev. A 43, 6247 (1991); (b) 43, 6258 (1991).

[6] R. Vyas and S. Singh, Phys. Rev. A 45, 8095 (1992); I. E. Lyublinskaya and R. Vyas, ibid. 48, 3966 (1993).

[7] S. Smart and S. Swain, Quantum Opt. 5, 75 (1993); Opt. Commun. 123, 310 (1996).

[8] N. Ph. Georgiades, E. S. Polzik, K. Edamatsu, H. J. Kimble, and A. S. Parkins, Phys. Rev. Lett. 75, 3426 (1995).

[9] Q. Turchette, N. Ph. Georgiades, C. Hood, H. J. Kimble, and A. S. Parkins, Phys. Rev. A 58, 4056 (1998).

[10] N. Ph. Georgiades, E. S. Polzik, and H. J. Kimble, Phys. Rev. A 55, 1605 (1997).

[11] N. Ph. Georgiades, E. S. Polzik, and H. J. Kimble (unpublished).

[12] N. Ph. Georgiades, E. S. Polzik, and H. J. Kimble, Opt. Lett. 21, 1688 (1996).

[13] M. J. Collet and R. Loudon, J. Opt. Soc. Am. B 4, 1525 (1987).

[14] C. Gardiner and A. S. Parkins, Phys. Rev. A 50, 1792 (1994).

[15] B. R. Mollow, Phys. Rev. 175, 1555 (1968).

[16] H. P. Yuen, Phys. Rev. A 13, 2226 (1976).

[17] H. J. Kimble, in Fundamental Systems in Quantum Optics, edited by J. Dalibard, J. M. Raimond, and J. Zinn-Justin (Elsevier, Amsterdam, 1992).

[18] H. J. Carmichael, An Open Systems Approach to Quantum Optics, Lecture Notes in Physics Vol. 18 (Springer-Verlag, Berlin, 1993), pp. 35-37.

[19] M. Sargent III, S. Ovadia, and M. H. Lu, Phys. Rev. A 32, 1596 (1985).

[20] A. W. Boone and S. Swain, Quantum Opt. 1, 27 (1989).

[21] K. J. McNeil and D. F. Walls, J. Phys. A 7, 617 (1974).

[22] T. Narseen and M. S. K. Razmi, Phys. Rev. A 46, 4161 (1992).

[23] Z. Chen and H. Freedhoff, J. Phys. B 24, 3373 (1991).

[24] B. Yurke, Phys. Rev. A 32, 311 (1985).

[25] C. M. Caves and B. L. Schumaker, in Quantum Optics IV, edited by J. D. Harvey and D. F. Walls (Springer-Verlag, Berlin, 1986), pp. 20-30.

[26] A. S. Zibrov, M. D. Lukin, D. E. Nikonov, L. Hollberg, M. O. Scully, V. L. Velichansky, and H. G. Robinson, Phys. Rev. Lett. 75, 1499 (1995); G. G. Padmabandu, G. R. Welch, I. N. Shubin, E. S. Fry, D. E. Nikonov, M. D. Lukin, and M. O. Scully, ibid. 76, 2053 (1996); S. Y. Zhu, L. M. Narducci, and M. O. Scully, Phys. Rev. A 52, 4791 (1995); C. Mavroyannis, ibid. 46, R6785 (1992).

[27] A. Kuzmich, K. Mølmer, and E. S. Polzik, Phys. Rev. Lett. 79, L1782 (1997).

[28] Z. Ficek, W. S. Smyth, and S. Swain, Phys. Rev. A 52, 4126 (1995); P. Zhou and S. Swain, ibid. 55, 772 (1997); P. Zhou and S. Swain, Opt. Commun. 134, 127 (1997); Z. Ficek, W. S. Smyth, and S. Swain, ibid. 110, 555 (1994). 